THE ASTROPHYSICAL JOURNAL, 441:689-701, 1995 March 10

(C) 1995. The American Astronomical Society. All rights reserved. Printed in U.S.A.

\title{
A MOLECULAR LINE STUDY OF NGC 1333/IRAS 4
}

\author{
Geoffrey A. Blake, ${ }^{1}$ Göran Sandell,${ }^{2}$ Ewine F. van Dishoeck, ${ }^{3}$ \\ T. D. Groesbeck, ${ }^{4}$ LeE G. Mundy,${ }^{5}$ AND Colin Aspin ${ }^{2}$ \\ Received 1994 June 16; accepted 1994 September 22
}

\begin{abstract}
Molecular line surveys and fully sampled spectral line maps at 1.3 and $0.87 \mathrm{~mm}$ are used to examine the physical and chemical characteristics of the extreme Class I sources IRAS 4A and 4B in the L1450/NGC 1333 molecular cloud complex. A very well collimated, jetlike molecular outflow emanates from IRAS 4A, with a dynamical age of a few thousand years. Symmetric, clumpy structure along the outflow lobes suggests that there is considerable variability in the mass-loss rate or wind velocity even at this young age. Molecular emission lines toward IRAS 4A and 4B are observed to be weak in the velocity range corresponding to quiescent material surrounding the young stellar objects (YSOs). Depletion factors of 10-20 are observed for all molecules, including $\mathrm{CO}$, even for very conservative mass estimates from the measured millimeter and submillimeter dust continuum. However, abundances scaled with respect to $\mathrm{CO}$ are similar to other dark molecular cloud cores. Such depletions could be mimicked by high dust optical depths or increased grain emissivities at the observing frequencies of 230 and $345 \mathrm{GHz}$, but the millimeter and submillimeter spectral energy distributions suggest that this is unlikely over the single-dish size scales of 5000-10,000 AU.

Dense, outflowing gas is found to be kinematically, but not spatially, distinct from the quiescent material on these size scales. If $\mathrm{CO}$ is used as a chemical standard for the high-velocity gas, we find substantial enhancements in the abundances of several molecules in outflowing material, most notably $\mathrm{CS}, \mathrm{SiO}$, and $\mathrm{CH}_{3} \mathrm{OH}$. The $\mathrm{SiO}$ emission is kinematically well displaced from the bulk cloud velocity and likely arises from directly shocked material. As is the case for $\mathrm{CO}$, however, the outflow features from more volatile species are centered near the cloud velocity and are often characterized by quite low rotational temperatures. We suggest that grain-grain collisions induced by velocity shear zones surrounding the outflow axes transiently desorb the grain mantles, resulting in large abundance enhancements of selected species. Similar results have recently been obtained in several other low-mass YSOs, where the outflowing gas is often both kinematically and spatially distinct, and are illustrative of the ability of accretion and outflow processes to simultaneously modify the composition of the gas and dust surrounding young stars.
\end{abstract}

Subject headings: ISM: abundances - ISM : individual (NGC 1333) - ISM: jets and outflows -

ISM: molecules - stars: formation

\section{INTRODUCTION}

Molecular clouds have been the objects of intense scrutiny for over two decades. Their role as stellar nurseries is beyond question, and the combination of rapidly improving observational tools at centimeter to near-infrared wavelengths and increasingly sophisticated theory has outlined, for the first time, the physical processes associated with the formation of Sun-like stars (for a suite of wide ranging reviews, see Levy \& Lunine 1993). Despite this rapid progress, much remains to be understood.

For example, heating of the gas and dust immediately surrounding young stellar objects (YSOs) produces dramatically different spectral energy distributions (SEDs) as a function of evolutionary state. This difference has been used to classify YSOs in a general sense (Lada 1991), but the origin of this emission is still controversial. The broad SEDs of T Tauri stars have typically been modeled as emission from centrifugally supported accretion disks (Adams, Lada, \& Shu 1987; Beck-

\footnotetext{
${ }^{1}$ Division of Geological and Planetary Sciences, 170-25, California Institute of Technology, Pasadena, CA 91125.

2 Joint Astronomy Center, 660 N. A'Ohoku Place, Hilo, HI 96720.

3 Leiden Observatory, P.O. Box 9513, 2300 RA Leiden, The Netherlands.

${ }^{4}$ Division of Physics, Mathematics and Astronomy, 320-47, California Institute of Technology, Pasadena, CA 91125.

5 Department of Astronomy, University of Maryland, College Park, MD 20742.
}

with et al. 1990), but recent reinterpretations have suggested that many of these objects are "protostellar" in the sense that they still support infall envelopes that provide significant infrared emission along with accretion rates of nearly $10^{-6} M_{\odot}$ (Kenyon, Calvet, \& Hartmann 1993; Calvet et al. 1994).

Accordingly, studies of truly protostellar sources, that is, sources which have yet to acquire the bulk of their final mass and derive most of their luminosity from accretion rather than nuclear fusion, would add much to our understanding of the birth of stars. The most obvious YSO signature in molecular clouds is not that of infall, but rather high velocity outflowing gas (see Fukui et al. 1993). These initially puzzling observations are now accepted as an integral phase of YSO evolution, and recent theories of low-mass star formation involve an extended phase of both accretion and outflow (Shu et al. 1993). Within this more general framework, a detailed understanding of the chemical composition and evolution of YSOs is of interest not only for its own sake, but also for the insight it may bring into how stars are born and the potentially dramatic influence chemistry may have on star formation rates and stellar mass distributions. For example, the fractional ionization of the gas, a critical parameter in magnetically mediated collapse models, is controlled directly by the chemical composition of molecular cloud cores (Hartquist et al. 1993).

The rich and diverse range of chemical species discovered in molecular clouds makes it possible, in principle, to derive not 
only the physical state of gas and dust in YSOs, but also to place constraints on the energetic processes and timescales associated with stellar birth if sufficiently distinct chemical signposts can be found. However, the observational uncertainties associated with objects in which both outflow and infall motions may simultaneously alter the chemical composition of YSOs are large. This, coupled with poorly characterized chemical and physical processes such as gas-grain interactions and grain mantle kinetics, renders the chemical classification of star-forming molecular cloud cores a difficult task (d'Hendecourt, Allamandola, \& Greenberg 1985; Brown \& Charnley 1990; Rawlings et al. 1992; Caselli, Hasegawa, \& Herbst 1993; Shalabiea \& Greenberg 1995).

Motivated by these concerns, we have begun a general survey of the chemistry associated with deeply embedded YSOs that cover a wide range of luminosities, but with an emphasis on regions suspected to be in the process of forming roughly solar mass stars (McMullin et al. 1994b; Helmich et al. 1994; Blake et al. 1994). The nearby NGC 1333 region, embedded in the L1450 molecular cloud complex, provides an excellent example of an active site of low-mass star formation. One of the more remarkable objects recently discovered in this cloud is designated NGC 1333/IRAS 4. It is a so-called extreme Class I (Lada 1991) or Class 0 (André \& Montmerle 1994) YSO, several of which have now been discovered. All possess dense envelopes surrounding the young stars which power molecular outflows. Those most similar IRAS 4, e.g., IRAS 16293-2422 and VLA 1623 (Mundy et al. 1992; André, Ward Thompson, \& Barsony 1993), can only be observed at far-infrared and longer wavelengths and possess visual extinctions of several hundred to a thousand magnitudes. These properties suggest that such sources are quite young, but inclination effects can greatly impact the estimated evolutionary state (Lada 1991; Mundy et al. 1992).

Continuum observations by Sandell et al. (1991) reveal NGC 1333/IRAS 4 to be extraordinarily massive and dense. Unresolved in IRAS observations (Jennings et al. 1987), IRAS 4 is suspected to be a wide binary system, with the two components having a projected separation of $\sim 30^{\prime \prime}$ at position angle $\sim 135^{\circ}$ (hereafter labeled IRAS 4A and 4B as in Sandell et al. 1991). Recent estimates of the distance to NGC 1333 have suggested it may be as close as $220 \mathrm{pc}$ (Cernis 1990) but note that there is a rapid increase in distance across the cloud (to $\sim 500 \mathrm{pc}$ ) as one moves to the east. We follow Herbig \& Jones (1983) for consistency with earlier work and adopt a distance of $350 \mathrm{pc}$ but also present, where appropriate, the changes required for the closer distance. At $350 \mathrm{pc}$, the gas and dust masses derived from the submillimeter continuum data are 9 and $4 M_{\odot}$, respectively (which drop to 3.5 and $1.5 M_{\odot}$ at $220 \mathrm{pc}$ ), with minimum beam-averaged gas densities of $9 \times 10^{9}$ and $9 \times 10^{8}$ $\mathrm{H}_{2}$ molecules $\mathrm{cm}^{-3}$ (Sandell et al. 1991). More extended mapping reveals additional mass at larger scales but lower physical densities, consistent with the conditions expected in star-forming molecular cloud cores (Knee et al. 1995).

We present here molecular line observations of IRAS 4A/4B which delineate the physical and chemical structures around these YSOs on the arcminute to 15 arcsec scale. In particular, we present fully sampled maps of the high- $J$ CO and CS emission from these objects along with molecular line surveys which probe the velocity fields, physical conditions, and molecular abundances of dense gas adjacent to the young stars. From these observations we construct a model that is used to examine the roles of processes such as gas-phase chemistry, grain mantle depletion, infall, and molecular outflows in establishing the observational morphology and chemical composition of extreme Class I YSOs.

\section{OBSERVATIONS}

Spectral lines between 200 and $400 \mathrm{GHz}$ from 10 different species and their isotopic variations were detected with the Caltech Submillimeter Observatory $(\mathrm{CSO})^{6} 10.4 \mathrm{~m}$ and the James Clerk Maxwell (JCMT) ${ }^{7} 15$ m telescopes. Upper limits were acquired for many other species. At the CSO, the facility $345 \mathrm{GHz}$ SIS receiver was coupled to the facility $500 \mathrm{MHz}$ and $50 \mathrm{MHz}$ bandwidth accousto-optical spectrometer (AOS) back ends which provide nominal resolutions of $1.0 \mathrm{MHz}$ and 0.1 $\mathrm{MHz}$. The actual resolution of the $500 \mathrm{MHz}$ AOS was often 2-3 times lower, and for the most important transitions the following line fits use only the $50 \mathrm{MHz}$ AOS data. The bulk of the CSO data were acquired in a single run during 1992 January. JCMT spectra were acquired in the 230 and $345 \mathrm{GHz}$ windows between 1992 February and 1993 December with receivers $\mathrm{A} 1, \mathrm{~A} 2$, and $\mathrm{B} 3 \mathrm{i}$. Most of the mapping data described below were acquired with the JCMT AOS, while the majority of the molecular emission line survey results were obtained with the Digital Autocorrelation Spectrometer (DAS).

The chopper wheel method (Kutner \& Ulich 1981) was used to provide emission-line strengths on the $T_{A}^{*}$ scale, which have then been converted into main-beam brightness temperatures, $T_{\mathrm{MB}}=T_{A}^{*} / \eta_{\mathrm{MB}}$. The CSO $\eta_{\mathrm{MB}}(345 \mathrm{GHz})$ is 0.60 , while the JCMT efficiencies range from $\eta_{\mathrm{MB}}(230 \mathrm{GHz})=0.65-0.70$ to $\eta_{\mathrm{MB}}(345 \mathrm{GHz})=0.52$ (1992 February). We estimate that the absolute calibration is accurate to within $\sim 30 \%$, but the relative calibration within each data set should be better.

The observed, diffraction-limited beam sizes for the two telescopes are $30^{\prime \prime}$ (CSO) and 21" (JCMT) at $230 \mathrm{GHz}$, decreasing to $20^{\prime \prime}$ and $14^{\prime \prime}$ at $345 \mathrm{GHz}$, respectively. These beam sizes correspond to linear sizes of 5000-10,000 AU at $350 \mathrm{pc}$. The similarity of the CSO $345 \mathrm{GHz}$ beam size and that of the JCMT at $230 \mathrm{GHz}$ provides a very well matched set of data with which to examine chemical abundances. Owing to the weakness of the emission from IRAS 4, complete molecular line surveys in the 1.3 and $0.87 \mathrm{~mm}$ atmospheric windows are not currently feasible. Selected transitions were therefore measured which probe a wide range of physical conditions and chemical processing.

In addition to the spectral line surveys, the JCMT was used to acquire maps of the $\mathrm{CO} J=3 \rightarrow 2$ and $\mathrm{CS} J=7 \rightarrow 6$ transitions near $345 \mathrm{GHz}$ as well as the CS $J=5 \rightarrow 4$ transition at $245 \mathrm{GHz}$. Several components of the methanol $J_{K}=5_{K} \rightarrow 4_{K}$ a-type band were mapped simultaneously with the CS $5 \rightarrow 4$ emission by placing them in the opposite receiver sideband. A preliminary discussion of these images may be found in Sandell \& Aspin (1993); a detailed description of the observing procedures will be presented elsewhere (Knee et al. 1995). These maps have been used to select regions free of emission for the reference beams used in the line survey measurements of lowexcitation species (e.g., ${ }^{13} \mathrm{CO}, \mathrm{C}^{18} \mathrm{O}$ ), and cover a region $5^{\prime}$ by

\footnotetext{
${ }^{6}$ The Caltech Submillimeter Observatory is operated by the California Institute of Technology under funding from the US National Science Foundation (AST 93-13929).

7 The James Clerk Maxwell Telescope is operated by the Royal Observatory, Edinburgh, on behalf of the UK Science and Engineering Research Council, the Netherlands Organization for Pure Research, and the National Research Council of Canada.
} 
$10^{\prime}$ in size at half-beam sampling intervals. From the compact appearance of the CS $7 \rightarrow 6$ emission as well as small maps of other species, it was found that measurements of highexcitation tracers could be acquired with azimuthal beam

I! switching by only a few arcminutes, thereby improving the observing efficiency and baselines considerably. Pointing was regularly monitored with planets and nearby secondary calibrators; errors are expected to be $\leq 5^{\prime \prime}$.

\section{RESULTS}

Table 1 presents a listing of the species and transitions detected in the molecular line surveys of IRAS 4A and 4B. Upper limits for species such as $\mathrm{SO}_{2}, \mathrm{HNCO}$, etc., are typically $T_{\mathrm{MB}} \leq 0.2 K(2 \sigma)$. The spectral profiles of the strongest emission lines, such as the CO $J=3 \rightarrow 2$ and CS $J=7 \rightarrow 6$ transitions presented in Figure 1, show all of the "classic" features associated with YSOs. Self-absorbed narrow components in the $\mathrm{CO}$ lines and narrow emission-line components $(\Delta V \sim 1-2$ $\mathrm{km} \mathrm{s}^{-1}$ ) in the CS transitions trace the quiescent molecular cloud core and envelope from which the young stars are accreting mass, while the high-velocity wings $\left(\Delta V \geq 10-15 \mathrm{~km} \mathrm{~s}^{-1}\right)$ on these features are formed from supersonic, outflowing winds. The relative amounts of material in these two reservoirs can be judged from optically thin tracers such as the ${ }^{13} \mathrm{CO}$ $J=3 \rightarrow 2$ transition also shown in Figure 1. The nearly identical LSR velocities of these lines toward IRAS 4A and 4B establish the relative proximity of the YSOs, but the small orbital velocity of the putative wide-binary system (Sandell et al. 1991) cannot be cleanly separated from other large scale cloud motions with the current data.

The fact that no wings are seen in the ${ }^{13} \mathrm{CO}$ transitions illustrates that the quiescent material includes substantially more mass than the high-velocity gas. As Figure 2 shows, commonly used molecular tracers sample the quiescent and highvelocity material with varying degrees of selectivity owing to their differing excitation requirements and chemical reactivity. These spectra also show the importance of high spectral resolution and high signal-to-noise ratio $(\mathrm{S} / \mathrm{N})$ observations,

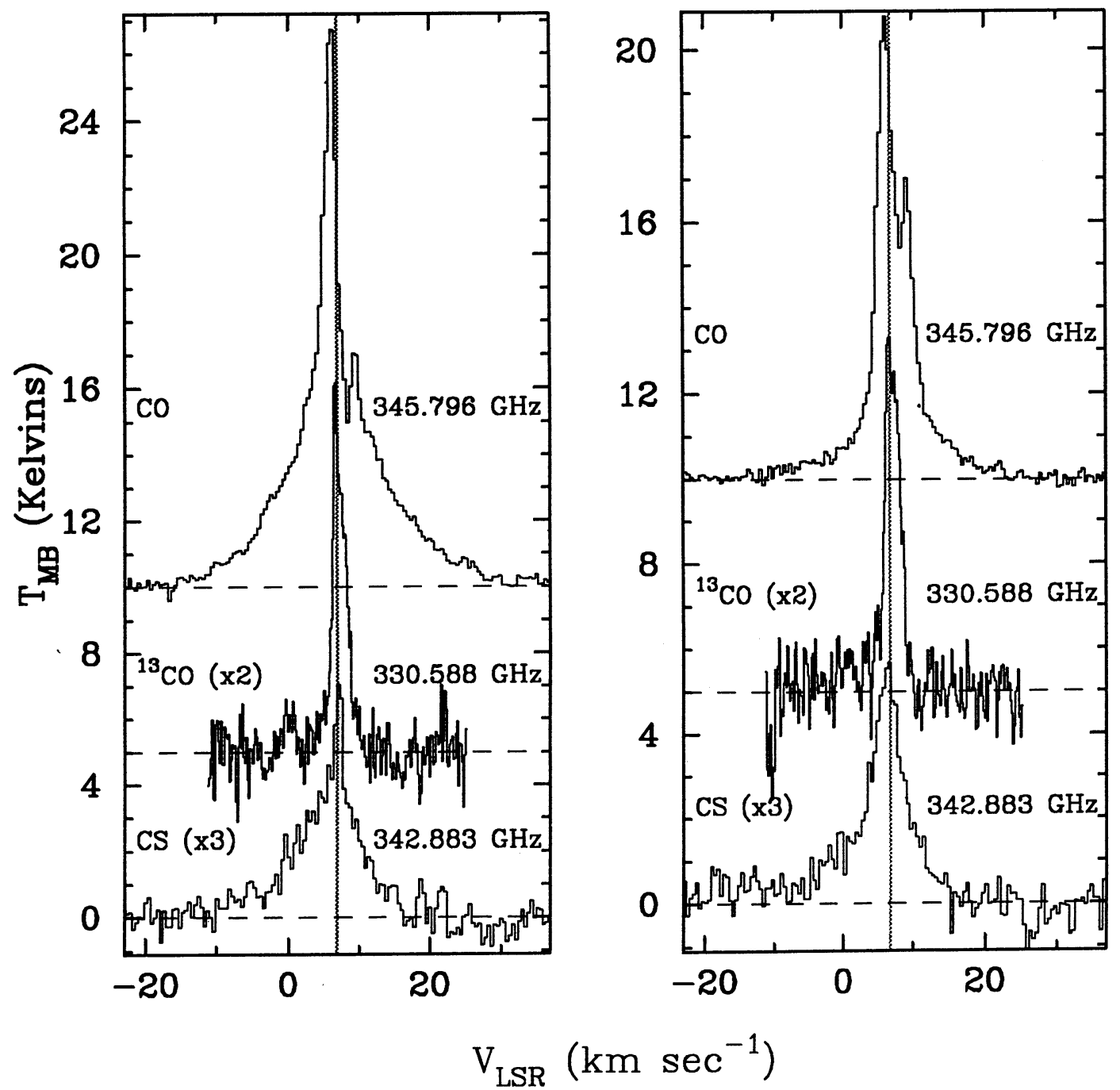

FIG. 1.-JCMT spectra of the CO $J=3 \rightarrow 2$ and CS $J=7 \rightarrow 6$ transitions and CSO spectra of the ${ }^{13} \mathrm{CO} J=3 \rightarrow 2$ transitions toward IRAS 4 A (left) [R.A. $(1950)=03^{\mathrm{h}} 26^{\mathrm{m}} 04^{\mathrm{s}} 96$, decl. $(1950)=31^{\circ} 03^{\prime} 13^{\prime \prime} 1$ ] and IRAS 4B (right) [R.A. $(1950)=03^{\mathrm{h}} 26^{\mathrm{m}} 06^{\mathrm{s}} .55$, decl. $(1950)=31^{\circ} 02^{\prime} 49^{\prime \prime}$. 5 . The strong wings on the parent isotopomer transitions result from dense, outflowing gas. The narrower line widths of the ${ }^{13} \mathrm{CO}$ transitions illustrate the low column density of higher velocity width outflowing material as compared to the quiescent core. The grey vertical line in this and subsequent figures marks the systemic velocity of $6.7 \mathrm{~km} \mathrm{~s} \mathrm{~s}^{-1}$ as determined by $\mathrm{CSO}$ high $\mathrm{S} / \mathrm{N}$ observations of the $\mathrm{C}^{18} \mathrm{O} J=3 \rightarrow 2$ transition. 
TABLE 1

ObServed Transitions, Diatomic and Linear Molecules

\begin{tabular}{|c|c|c|c|c|c|c|c|c|c|c|c|}
\hline \multirow[b]{2}{*}{ MoleCule } & \multirow[b]{2}{*}{ LINE } & \multirow[b]{2}{*}{$\begin{array}{c}\text { FREQUENCY } \\
(\mathrm{MHz})\end{array}$} & \multicolumn{4}{|c|}{ IRAS 4A } & \multicolumn{4}{|c|}{ IRAS 4B } & \multirow[b]{2}{*}{ Telescope } \\
\hline & & & $\underset{\left(\mathrm{K} \mathrm{km} \mathrm{s}^{-1}\right)}{T_{\mathrm{MB}} d V}$ & $\begin{array}{l}T_{\mathrm{MB}} \\
(\mathrm{K})\end{array}$ & $\begin{array}{c}\Delta V \\
\left(\mathrm{~km} \mathrm{~s}^{-1}\right)\end{array}$ & $\frac{V_{\mathrm{LSR}}}{\left(\mathrm{km} \mathrm{s}^{-1}\right)}$ & $\underset{\left(\mathrm{K} \mathrm{km} \mathrm{s}^{-1}\right)}{T_{\mathrm{MB}} d V}$ & $\begin{array}{l}T_{\mathrm{MB}} \\
(\mathrm{K})\end{array}$ & $\begin{array}{c}\Delta V \\
\left(\mathrm{~km} \mathrm{~s}^{-1}\right)\end{array}$ & $\begin{array}{c}V_{\mathrm{LSR}} \\
\left(\mathrm{km} \mathrm{s}^{-1}\right)\end{array}$ & \\
\hline \multirow[t]{2}{*}{$\mathrm{CO} \ldots \ldots \ldots \ldots$} & \multirow[t]{2}{*}{$3-2$} & \multirow[t]{2}{*}{345796.0} & 135.4 & & $\ldots$ & $\ldots$ & 64.20 & & $\ldots$ & $\ldots$ & JCMT AOSC \\
\hline & & & 83.42 & 13.3 & $\ldots$ & 6.8 & 47.18 & 9.6 & $\ldots$ & 6.6 & CSO $50 \mathrm{MHz}$ \\
\hline${ }^{13} \mathrm{CO} \ldots \ldots \ldots \ldots$ & $3-2$ & 330588.1 & 15.96 & 4.92 & 3.05 & 6.1 & 9.80 & 3.45 & 2.67 & 6.2 & CSO $50 \mathrm{MHz}$ \\
\hline \multirow[t]{2}{*}{$\mathrm{C}^{18} \mathrm{O} \ldots \ldots \ldots$} & $2-1$ & 219560.3 & 5.00 & 2.33 & 2.00 & 7.1 & 4.67 & 2.00 & 2.20 & 7.1 & JCMT DAS \\
\hline & $3-2$ & 329331.0 & 4.87 & 1.82 & 2.52 & 6.7 & 3.29 & 1.27 & 2.44 & 6.6 & CSO $500 \mathrm{MHz}$ \\
\hline $\mathrm{C}^{17} \mathrm{O} \ldots \ldots \ldots$ & $3-2$ & 337061.1 & 1.01 & 0.40 & 2.37 & 6.5 & 0.80 & 0.35 & 2.16 & 6.7 & CSO $50 \mathrm{MHz}$ \\
\hline \multirow{6}{*}{$\mathrm{CS} \ldots \ldots \ldots \ldots \ldots$} & \multirow{2}{*}{$5-4$} & 244935.6 & 3.11 & 2.92 & 1.00 & 6.7 & 2.45 & 1.54 & 1.50 & 6.8 & JCMT DAS \\
\hline & & & 15.76 & 1.38 & 10.7 & 5.7 & 10.31 & 1.08 & 9.00 & 7.6 & JCMT DAS \\
\hline & $7-6$ & 342882.0 & 1.35 & 1.15 & 1.10 & 7.1 & 2.66 & 0.96 & 2.60 & 7.6 & JCMT AOSC \\
\hline & & & 13.87 & 1.15 & 11.3 & 5.6 & 11.25 & 0.96 & 11.0 & 6.9 & JCMT AOSC \\
\hline & $7-6$ & 342882.0 & 1.22 & 1.18 & 0.97 & 6.8 & 2.85 & 0.79 & 3.39 & 6.6 & CSO $50 \mathrm{MHz}(4 \mathrm{~A})$ \\
\hline & & & 11.75 & 1.15 & 9.60 & 7.0 & 9.25 & 0.72 & 12.2 & 7.4 & CSO $500 \mathrm{MHz}(4 \mathrm{~B})$ \\
\hline$C^{34} S \ldots \ldots \ldots$ & $5-4$ & 241016.2 & 1.00 & 0.45 & 2.11 & 6.9 & $\ldots$ & $\ldots$ & $\ldots$ & $\ldots$ & JCMT DAS \\
\hline & $7-6$ & 337396.7 & $\ldots$ & $<0.13$ & $\ldots$ & $\ldots$ & $\ldots$ & $\ldots$ & $\ldots$ & $\ldots$ & CSO $500 \mathrm{MHz}$ \\
\hline $\mathrm{SiO} \ldots \ldots \ldots \ldots$ & $5-4$ & 217104.9 & 2.51 & 0.20 & 11.5 & -0.5 & $\ldots$ & $\ldots$ & $\ldots$ & $\ldots$ & JCMT DAS \\
\hline SO ........... & $6_{5}-5_{4}$ & 219949.4 & 3.54 & 1.96 & 1.70 & 6.7 & $\ldots$ & $\ldots$ & $\ldots$ & $\ldots$ & JCMT DAS \\
\hline & & & 5.77 & 0.40 & 13.6 & 3.1 & $\ldots$ & $\ldots$ & $\ldots$ & $\ldots$ & JCMT DAS \\
\hline HCN .......... & $4-3$ & 354505.5 & 0.67 & 0.48 & 1.33 & 6.6 & 3.28 & 0.70 & 4.41 & 5.6 & CSO $500 \mathrm{MHz}$ \\
\hline & & & 15.07 & 0.90 & 15.8 & 6.9 & 8.93 & 0.48 & 17.6 & 7.0 & CSO $500 \mathrm{MHz}$ \\
\hline DCN .......... & $3-2$ & 217238.6 & 0.86 & 0.34 & 2.40 & 7.2 & $\ldots$ & $\ldots$ & $\ldots$ & $\ldots$ & JCMT DAS \\
\hline HNC .......... & 4-3 & 362629.9 & 3.42 & 0.79 & 4.07 & 6.9 & 2.10 & 0.75 & 2.63 & 7.1 & CSO $500 \mathrm{MHz}$ \\
\hline $\mathrm{HCO}^{+} \ldots \ldots \ldots$ & $4-3$ & 356734.3 & 3.72 & 3.58 & 0.98 & 6.5 & 5.39 & 3.33 & 1.52 & 6.5 & CSO $50 \mathrm{MHz}$ \\
\hline & & & 5.47 & 1.08 & 4.76 & 7.1 & $\ldots$ & $\ldots$ & $\ldots$ & $\ldots$ & CSO $50 \mathrm{MHz}$ \\
\hline $\mathrm{H}^{13} \mathrm{CO}^{+} \ldots \ldots$ & $3-2$ & 260255.5 & 1.27 & 0.95 & 1.26 & $\ldots$ & $\ldots$ & $\ldots$ & $\ldots$ & $\ldots$ & JCMT DAS \\
\hline & $4-3$ & 344000.0 & 0.86 & 0.67 & 1.21 & 6.9 & $\ldots$ & $<0.1$ & $\ldots$ & $\ldots$ & CSO $500 \mathrm{MHz}$ \\
\hline $\mathrm{DCO}^{+} \ldots \ldots \ldots$ & $3-2$ & 216112.6 & 2.43 & $\ldots$ & 1.5 & $\ldots$ & $\ldots$ & $\ldots$ & $\ldots$ & $\ldots$ & CSO $500 \mathrm{MHz}$ \\
\hline & $5-4$ & 360170.0 & 0.64 & 0.38 & 1.56 & 6.9 & $\ldots$ & $<0.2$ & $\ldots$ & $\ldots$ & CSO $50 \mathrm{MHz}$ \\
\hline $\mathrm{N}_{2} \mathrm{H}^{+} \ldots \ldots \ldots$ & 4-3 & 372672.5 & 5.64 & $\ldots$ & 1.5 & $\ldots$ & $\ldots$ & $\ldots$ & $\ldots$ & $\ldots$ & CSO $500 \mathrm{MHz}$ \\
\hline $\mathrm{CH}_{3} \mathrm{OH} \ldots \ldots$ & $4_{2}-3_{1} E^{+}$ & 218440.0 & 1.79 & 0.38 & 4.40 & 6.8 & 1.34 & 0.57 & 2.50 & 7.2 & JCMT DAS \\
\hline & $5_{0}-4_{0} \mathrm{E}^{+}$ & 241700.2 & 4.35 & 0.72 & 5.68 & 6.8 & 2.94 & 0.75 & 3.68 & 7.0 & JCMT DAS \\
\hline & $5_{1}-4_{1} \mathrm{E}^{-}$ & 241767.2 & 3.22 & 1.44 & 2.10 & 7.3 & 5.06 & 1.40 & 3.40 & 7.5 & JCMT DAS \\
\hline & & & 6.72 & 0.78 & 8.10 & 3.7 & 0.60 & 0.12 & 4.60 & 6.8 & JCMT DAS \\
\hline & $5_{0}-4_{0} \mathrm{~A}^{+}$ & 241791.4 & 3.37 & 1.38 & 2.30 & 7.4 & 5.41 & 1.75 & 2.90 & 8.0 & JCMT DAS \\
\hline & & & 9.12 & 0.86 & 10.0 & 4.0 & 1.66 & 0.35 & 4.40 & 5.8 & JCMT DAS \\
\hline & $5_{4}-4_{4} A^{ \pm}$ & 241806.5 & $\ldots$ & $<0.09$ & $\ldots$ & $\ldots$ & $\ldots$ & $<0.11$ & $\ldots$ & $\ldots$ & JCMT AOSC \\
\hline & $5_{3}^{4}-4_{3}^{4} \mathrm{~A}^{ \pm}$ & 241833.0 & $\ldots$ & $<0.08$ & $\ldots$ & $\ldots$ & $\ldots$ & $<0.11$ & $\ldots$ & $\ldots$ & JCMT AOSC \\
\hline & $5_{2}-4_{2} \mathrm{~A}^{+}$ & 241842.3 & $\ldots$ & $<0.08$ & $\ldots$ & $\ldots$ & $\ldots$ & $\leq 0.26$ & $\ldots$ & 6.7 & JCMT AOSC \\
\hline & $5_{3}-4_{3} \mathrm{E}^{-}$ & 241852.4 & $\ldots$ & $<0.09$ & $\ldots$ & $\ldots$ & $\ldots$ & $<0.11$ & $\ldots$ & $\ldots$ & JCMT AOSC \\
\hline & $5_{1}-4_{1} \mathrm{E}^{+}$ & 241879.1 & 0.55 & 0.30 & 1.70 & 6.9 & 0.44 & 0.20 & 2.10 & 7.0 & JCMT DAS \\
\hline & & & 1.55 & 0.18 & 7.90 & 5.3 & 0.91 & 0.21 & 4.00 & 7.0 & JCMT DAS \\
\hline & $5_{2}-4_{2} \mathrm{~A}^{+}$ & 241887.7 & $\ldots$ & 0.29 & $\ldots$ & 6.1 & $\ldots$ & $<0.14$ & $\ldots$ & $\ldots$ & JCMT DAS \\
\hline & $5_{2}-4_{2} \mathrm{E}^{ \pm}$ & 241904.3 & 0.79 & 0.41 & 1.80 & 5.9 & 1.15 & 0.40 & 2.70 & 7.2 & JCMT DAS \\
\hline & & & 2.96 & 0.28 & 10.0 & 4.5 & 1.72 & 0.22 & 7.50 & 6.4 & JCMT DAS \\
\hline $\mathrm{H}_{2} \mathrm{CO} \ldots \ldots \ldots$ & $3_{03}-2_{02}$ & 218222.2 & 3.50 & 2.28 & 1.44 & 7.0 & 4.09 & 1.83 & 2.10 & 6.8 & JCMT DAS \\
\hline & & & 8.19 & 0.91 & 8.50 & 4.6 & 4.41 & 0.46 & 9.00 & 7.8 & JCMT DAS \\
\hline & $3_{22}-2_{21}$ & 218475.6 & 0.86 & 0.39 & 2.10 & 6.4 & 1.44 & 0.54 & 2.50 & 6.9 & JCMT DAS \\
\hline & & & 2.32 & 0.25 & 8.60 & 3.8 & $\ldots$ & $\ldots$ & $\ldots$ & $\ldots$ & JCMT DAS \\
\hline & $3_{21}-2_{20}$ & 218760.1 & 0.68 & 0.46 & 1.40 & 7.1 & 0.69 & 0.46 & 1.40 & 7.0 & JCMT DAS \\
\hline & & & 2.69 & 0.22 & 11.0 & 6.6 & 1.62 & 0.24 & 6.40 & 7.8 & JCMT DAS \\
\hline & $3_{12}-2_{11}$ & 225697.8 & 5.55 & 3.19 & 1.64 & 6.6 & 4.54 & 2.60 & 1.64 & 6.5 & JCMT DAS \\
\hline & & & 12.78 & 1.54 & 7.80 & 6.1 & 7.73 & 1.23 & 5.90 & 7.4 & JCMT DAS \\
\hline & $5_{15}-4_{14}$ & 351768.7 & 4.67 & 1.50 & 2.93 & 7.8 & 4.07 & 1.53 & 3.42 & 8.4 & CSO $500 \mathrm{MHz}$ \\
\hline & & & 16.23 & 1.27 & 12.0 & 8.4 & 8.02 & 0.66 & 11.4 & 8.5 & CSO $500 \mathrm{MHz}$ \\
\hline & $5_{05}-4_{04}$ & 362735.9 & 0.78 & 0.49 & 1.51 & 7.6 & 3.38 & 0.92 & 3.47 & 7.3 & CSO $500 \mathrm{MHz}$ \\
\hline & & & 5.77 & 0.91 & 5.96 & 8.5 & $\ldots$ & $\ldots$ & $\ldots$ & $\ldots$ & CSO $500 \mathrm{MHz}$ \\
\hline & $5_{24}-4_{23}$ & 363945.9 & 1.95 & 0.45 & 4.08 & 5.2 & 3.55 & 0.51 & 6.53 & 5.9 & CSO $500 \mathrm{MHz}$ \\
\hline & $5_{41 / 2}-4_{40 / 1}$ & 364102.8 & $\leq 0.65$ & $\leq 0.28$ & 2.17 & 5.2 & $\leq 1.90$ & $\leq 0.16$ & 10.9 & 7.8 & CSO $500 \mathrm{MHz}$ \\
\hline & $5_{33}-432$ & 364275.2 & $\leq 3.15$ & $\leq 0.43$ & 6.89 & 8.1 & $\leq 2.80$ & $\leq 0.43$ & 6.14 & 7.5 & CSO $500 \mathrm{MHz}$ \\
\hline & & 364289.0 & $\leq 5.67$ & $\leq 0.43$ & 12.4 & 5.2 & $\leq 2.72$ & $\leq 0.35$ & 7.35 & 7.7 & CSO $500 \mathrm{MHz}$ \\
\hline & & 365363.4 & 1.29 & 0.41 & 2.95 & 6.8 & 1.00 & 0.30 & 2.13 & 7.0 & CSO $500 \mathrm{MHz}$ \\
\hline $\mathrm{H}_{2}^{13} \mathrm{CO}$ & $3_{12}-2_{11}$ & 219908.5 & 0.86 & 0.17 & 4.80 & 7.2 & $\ldots$ & $\ldots$ & $\ldots$ & $\ldots$ & JCMT DAS \\
\hline
\end{tabular}




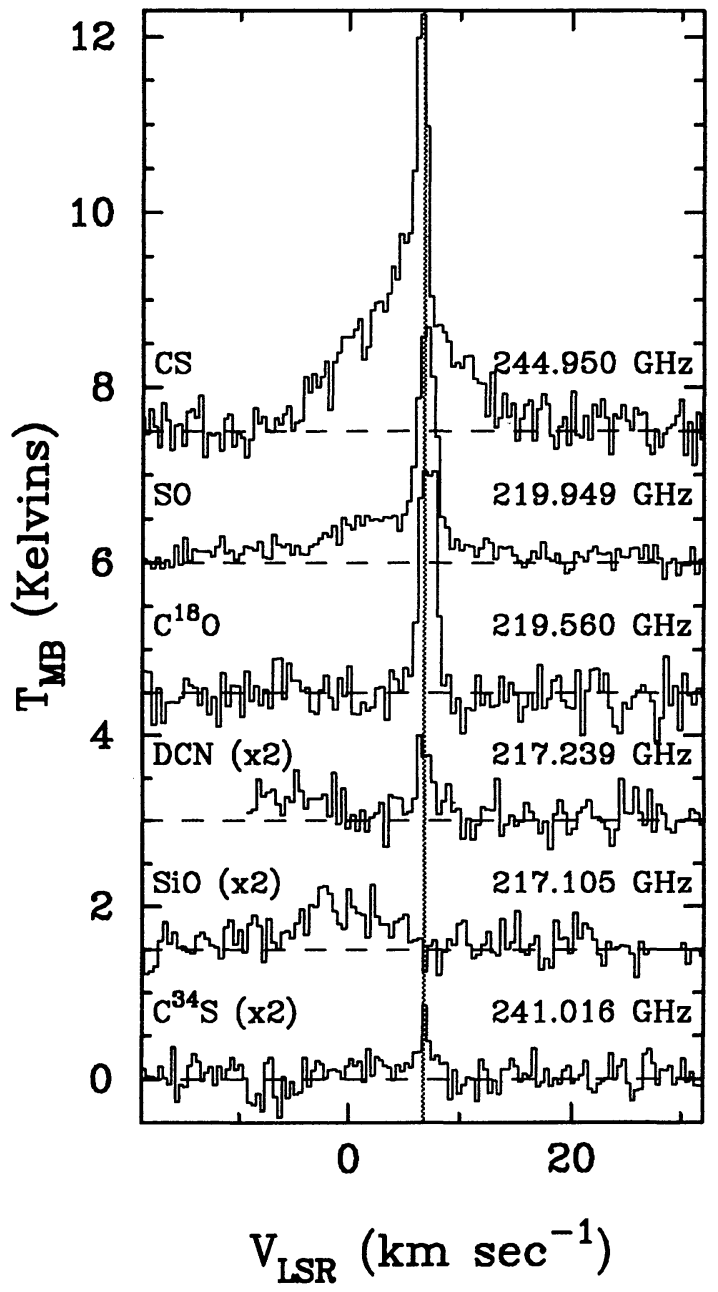

FIG. 2.-JCMT spectra of a variety of molecular transitions in the $1.3 \mathrm{~mm}$ atmospheric window which illustrate the wide range of kinematic profiles observed toward IRAS $4 \mathrm{~A}$. Bulk mass tracers such as $\mathrm{C}^{18} \mathrm{O}$ have narrow profiles characteristic of the quiescent cores surrounding the YSOs, while tracers of high-energy processes such as sputtering (e.g., SiO) shown broad velocity profiles offset from the systemic velocity. Species which are present in both environments (CS, SO, etc.) often show line profiles with multiple components.

since the line shapes of the various species can provide direct clues to their origin. It must be kept in mind, however, that the decomposition of line profiles into two or more components can introduce significant analytical uncertainties unless the kinematic signatures are truly distinct, particularly if the lines are not well resolved.

Before turning our attention to a detailed examination of the line surveys, we first describe the large-scale structure of the molecular outflow(s) from IRAS 4A and 4B.

\subsection{The Molecular Outflows from IRAS $4 A$ and $4 B$}

In Figures 3 and 4 we present an overview of the results from the JCMT CO $3 \rightarrow 2 / \mathrm{CS} 7 \rightarrow 6$ imaging of the $\sim 4^{\prime} \times 4^{\prime}$ region surrounding IRAS 4A and 4B (Sandell \& Aspin 1993; Knee et al. 1995). In both figures the stars denote the positions of the YSOs as determined by interferometric dust continuum observations between 22.7 and $218.6 \mathrm{GHz}$ (Mundy et al. 1995). Immediately apparent in the $\mathrm{CO} 3 \rightarrow 2$ image is a well collimated molecular outflow centered on the continuum position

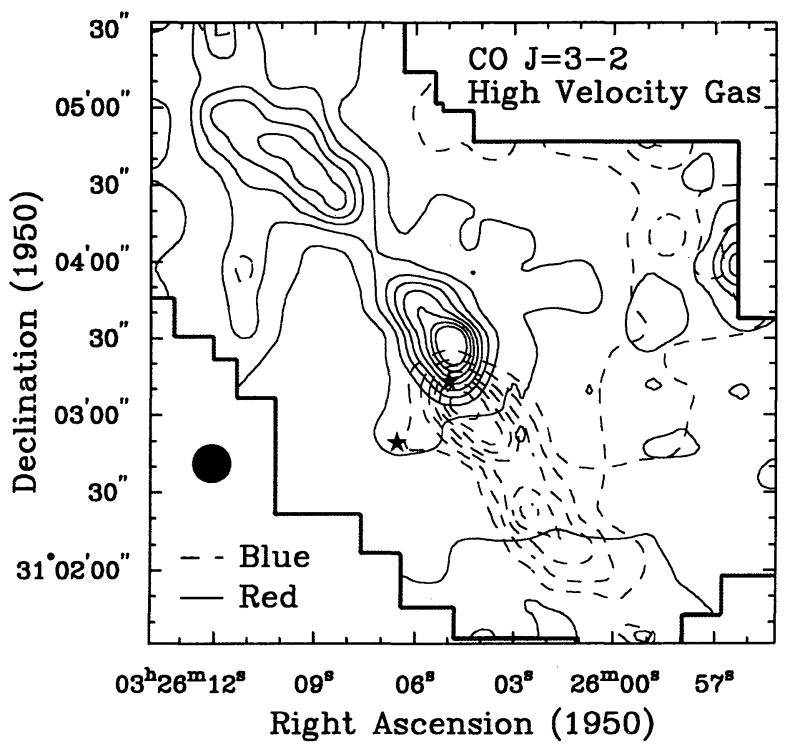

FIG. 3. -JCMT map of the CO $J=3 \rightarrow 2$ high velocity outflow in the vicinity of NGC 1333/IRAS 4. The solid contours depict emission to the red of the systemic velocity, while the dotted contours outline the limits of the blue wing. Stars mark the positions of IRAS $4 \mathrm{~A}$ and $4 \mathrm{~B}$ as deduced from highresolution millimeter-wave interferometric maps of the dust continuum emission (Mundy et al. 1995). Contour levels start at $2 \mathrm{~K} \mathrm{~km} \mathrm{~s}^{-1}$ and are spaced by $2 \mathrm{~K} \mathrm{~km} \mathrm{~s}^{-1}$.

of IRAS 4A, which extends over roughly $4^{\prime}$ tip-to-tip. A rather abrupt change of the position angle of the flow from approximately $45^{\circ}$ on the large scale to $0^{\circ}$ near IRAS $4 \mathrm{~A}$ is also clearly discernible. The $\mathrm{CO}$ lobes show considerable symmetric structure along their axes and are nearly mirror images of each other. Such symmetry is unlikely to be caused by external perturbations from the surrounding molecular cloud core and is suggestive of episodic variability in the mass-loss rate, wind velocity, or both. Some $100^{\prime \prime}-120^{\prime \prime}$ distant from IRAS 4A, the

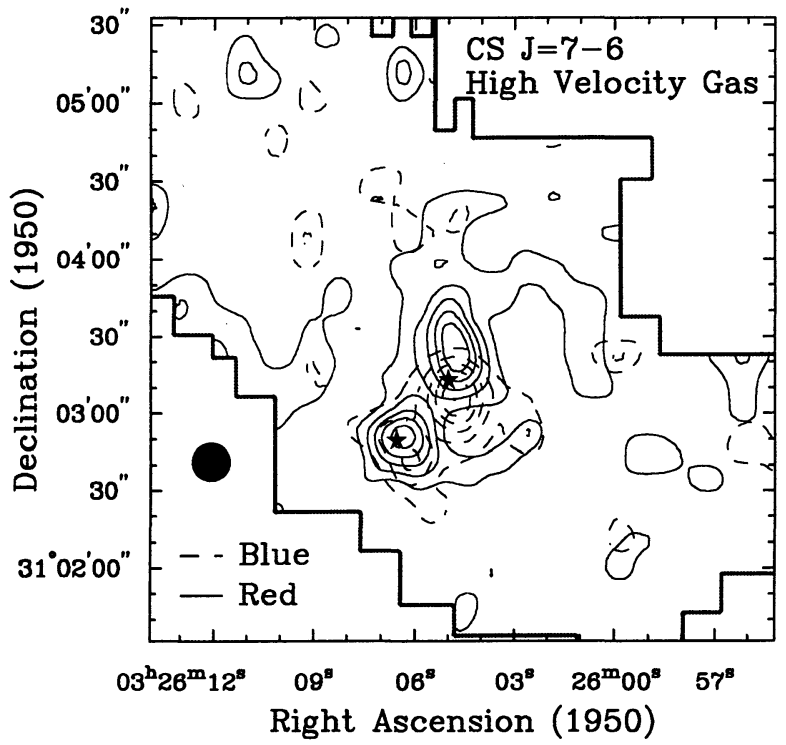

FIG. 4.-JCMT map of the CS $J=7 \rightarrow 6$ outflow, in which the solid contours again depict the red wing, while the dotted contours outline the limits of the blue wing. The very high density structure of the inner structure of the $\mathrm{CO}$ $3 \rightarrow 2$ map is clearly illustrated. Contour levels start at $0.3 \mathrm{~K} \mathrm{~km} \mathrm{~s}^{-1}$ and are spaced by $0.3 \mathrm{~K} \mathrm{~km} \mathrm{~s}^{-1}$ 
outermost clumps of the outflow are essentially unresolved along their minor axis and lead to collimation factors of 10 15-among the highest ever observed for molecular tracers of cold, outflowing gas from YSOs. These high collimation factors and relatively low wing velocities suggest that the outflow is viewed nearly in the plane of the sky, although there is insufficient information to make quantitative estimates of the system inclination angle.

Maps in high-density tracers (e.g., CS $5 \rightarrow 4$, CS $7 \rightarrow 6$ ) reveal only the innermost region of the flow from IRAS $4 A$, but the strong wings on these features demonstrate that densities in the outflowing material near the young stars are high, at least several $10^{6} \mathrm{~cm}^{-3}$. The flow around IRAS 4B, visible as highvelocity features on both the $\mathrm{CO}$ and $\mathrm{CS}$ lines in Figure 1, is most clearly delineated in CS line-wing emission. The absence of CS $7 \rightarrow 6$ emission in the lobes near the terminus of the IRAS 4A flow demonstrates that the gas is not nearly as dense or warm there. Position-velocity cuts along the axis of the IRAS 4A outflow reveal that the clumpy structure along the outflow axis (see Fig. 3) is induced more by variations in the velocity dispersion than by modulations in $\mathrm{CO}$ intensity.

The structure of the high-velocity $\mathrm{CO}$ outflowing gas is very similar to recent jet-driven models of molecular outflows (Masson \& Chernin 1993; Raga \& Cabrit 1993) and suggests that the outflow age is quite small. Crossing times of 10,000 $20,000 \mathrm{yr}$ are estimated for the observed $\mathrm{CO}$ velocity of $10-20$ $\mathrm{km} \mathrm{s}^{-1}$. The true outflow velocities are likely to be larger given the probable low inclination angles of these sources. Indeed, flow models in which a $100-300 \mathrm{~km} \mathrm{~s}^{-1}$ jet sweeps up mass to produce the observed outflow lobes suggest dynamical times of only $2000-5000 \mathrm{yr}$ at a distance of $350 \mathrm{pc}(1250-3000 \mathrm{yr}$ at 220 pc). Based on their extent, the high-density outflow structures nearest to IRAS $4 \mathrm{~A}$ and $4 \mathrm{~B}$ could be produced in just a few hundred years.

The observed ${ }^{12} \mathrm{CO} /{ }^{13} \mathrm{CO}$ ratio of $\geq 20$ in the line wings (see Fig. 1) limits the optical depth in the outflowing gas to $\tau\left({ }^{12} \mathrm{CO}\right) \leq 3$. Following Chernin \& Masson (1991) and the excitation results described below, we utilize an excitation temperature of $\sim 20 \mathrm{~K}$ for the overall outflow and derive a total momentum of $\sim 1.4 \mathrm{M}_{\odot} \mathrm{km} \mathrm{s}^{-1}$. For the ejected material closest to IRAS 4A and 4B the excitation temperature in highdensity tracers is found to be closer to $60 \mathrm{~K}$, which results in an IRAS 4A outflow mass and momentum of $0.12 M_{\odot}$ and 0.24 $M_{\odot} \mathrm{km} \mathrm{s}^{-1}$ over $20^{\prime \prime}$ scales, with the flow to the south (the blue lobe) being more extended and containing approximately twice the mass of the northern, or red, lobe (multiply the above by 0.4 for a distance of $220 \mathrm{pc}$ ).

These swept-up masses and momenta can easily be accounted for with the outflow models noted above, provided the "stellar jets" sustain velocities of $100-300 \mathrm{~km} \mathrm{~s}^{-1}$ at massloss rates of a few $10^{-6} M_{\odot}$. In such models, the wider opening angle of most observed CO outflows is due to a "wandering" of the jet axis with time, which causes the apparent dynamical age to be considerably shorter than the true age of the flow (Masson \& Chernin 1993). Thus, the highly collimated, symmetric character of the IRAS 4A CO outflow suggests that its true age should be close to the estimated dynamical timescale(s).

\subsection{Physical Conditions and Column Densities toward IRAS $4 A$ and $4 B$}

Provided the molecular line emission is optically thin and arises from levels that are in local thermodynamic equilibrium
(LTE), the following equation relates the integrated line strength, column density, and temperature:

$$
\frac{3 k \int T_{\mathrm{MB}} d V}{8 \pi^{3} \mu^{2} v S}=\frac{N_{u}}{g_{u}}=\frac{N_{T}}{Q\left(T_{\mathrm{ROT}}\right)} e^{-E_{u} / k T_{\mathrm{ROT}}} .
$$

Here $\mu, v$, and $S$ are the transition dipole moment, frequency, and line strength; $N_{T}$ is the total column density; $T_{R O T}$ is the LTE rotational temperature; $E_{u}$ is the upper state energy; and $Q\left(T_{\text {ROT }}\right)$ is the rotational partition function. The level degeneracies $g_{u}$, line strengths, and partition functions must be defined consistently, and for these analyses we have used an updated version of the JPL microwave, millimeter, and submillimeter spectral line catalog (Poynter \& Pickett 1984; Groesback 1994). As noted above, the matched beam sizes of the JCMT $230 \mathrm{GHz}$ and CSO $345 \mathrm{GHz}$ data render them less susceptible to uncertainties due to variations in source sizes and excitation gradients. In general, rotational temperatures in the $15-20 \mathrm{~K}$ range are found for species expected to be optically thin, such as $\mathrm{C}^{18} \mathrm{O}, \mathrm{H}^{13} \mathrm{CO}^{+}$, and $\mathrm{C}^{34} \mathrm{~S}$. Only for $\mathrm{CO}$, however, are the levels truly likely to approach those expected in LTE. For species with higher excitation requirements, the $T_{\text {ROT }}$ values form lower bounds to the true kinetic temperature.

Sufficiently numerous lines to provide well constrained estimates of the rotation temperature were obtained only for $\mathrm{H}_{2} \mathrm{CO}$ and $\mathrm{CH}_{3} \mathrm{OH}$. $T_{\mathrm{ROT}}$ values for the observed $\mathrm{CH}_{3} \mathrm{OH}$ transitions are only $15-17 \mathrm{~K}$ in both IRAS $4 \mathrm{~A}$ and $4 \mathrm{~B}$, while those for the $\mathrm{H}_{2} \mathrm{CO}$ lines are approximately $40-60 \mathrm{~K}$ if total integrated line intensities are used. The methanol transitions are observed within a single receiver passband, and as such the relative calibration of the emission strengths is very good. For the formaldehyde observations, many local oscillator settings are required as well as an accurate knowledge of intertelescope calibration. Accordingly, the error bars are substantially larger. Furthermore, in certain transitions there is a marked difference in the line widths of the quiescent, or "core" material, and that which participates in the outflow. By applying two-component Gaussian fits to the data, we can estimate core versus outflow emission parameters. The core $\mathrm{H}_{2} \mathrm{CO}$ fits give consistently colder rotational temperatures, $\sim 20-30 \mathrm{~K}$ for IRAS $4 \mathrm{~A}$ and $4 \mathrm{~B}$, while the wing fits for IRAS $4 \mathrm{~A}$ are consistent with $T_{\text {ROT }} \gtrsim 65-70 \mathrm{~K}$.

To make the best possible estimate of the true kinetic temperature and gas density, we have analyzed the observed line ratios with full statistical equilibrium/radiative transfer calculations. We have previously found that $\mathrm{CS}$ and $\mathrm{H}_{2} \mathrm{CO}$ are excellent complementary probes of gas conditions. Details of the calculational procedure may be found in Jansen, van Dishoeck, \& Black (1993) and van Dishoeck, Jansen, \& Phillips (1993). Figures 5 and 6 present the results for these two species, with Figure 5 concentrating on line ratios that are most sensitive to density, while Figure 6 presents those ratios that are most sensitive to temperature.

As Figures 5 and 6 illustrate, the core material is not only colder than the outflowing gas, as the rotation diagrams suggest, it is also slightly less dense. Best estimates for the physical conditions in these two regions are $T_{\mathrm{KIN}} \simeq 20-40 \mathrm{~K}$, $n_{\mathrm{H}_{2}} \simeq 2 \times 10^{6} \mathrm{~cm}^{-3}$ for the core and $T_{\mathrm{KIN}} \simeq 70-100 \mathrm{~K}, n_{\mathrm{H}_{2}} \simeq$ $5 \times 10^{6} \mathrm{~cm}^{-3}$ for the wing material near IRAS 4A. Estimates for the core material toward IRAS $4 B$ are similar, although the kinematic distinction between core and wing components is not nearly as clear. Similar density estimates for the core material are found from the line ratios of $\mathrm{H}^{13} \mathrm{CO}^{+}$and $\mathrm{DCO}^{+}$. 

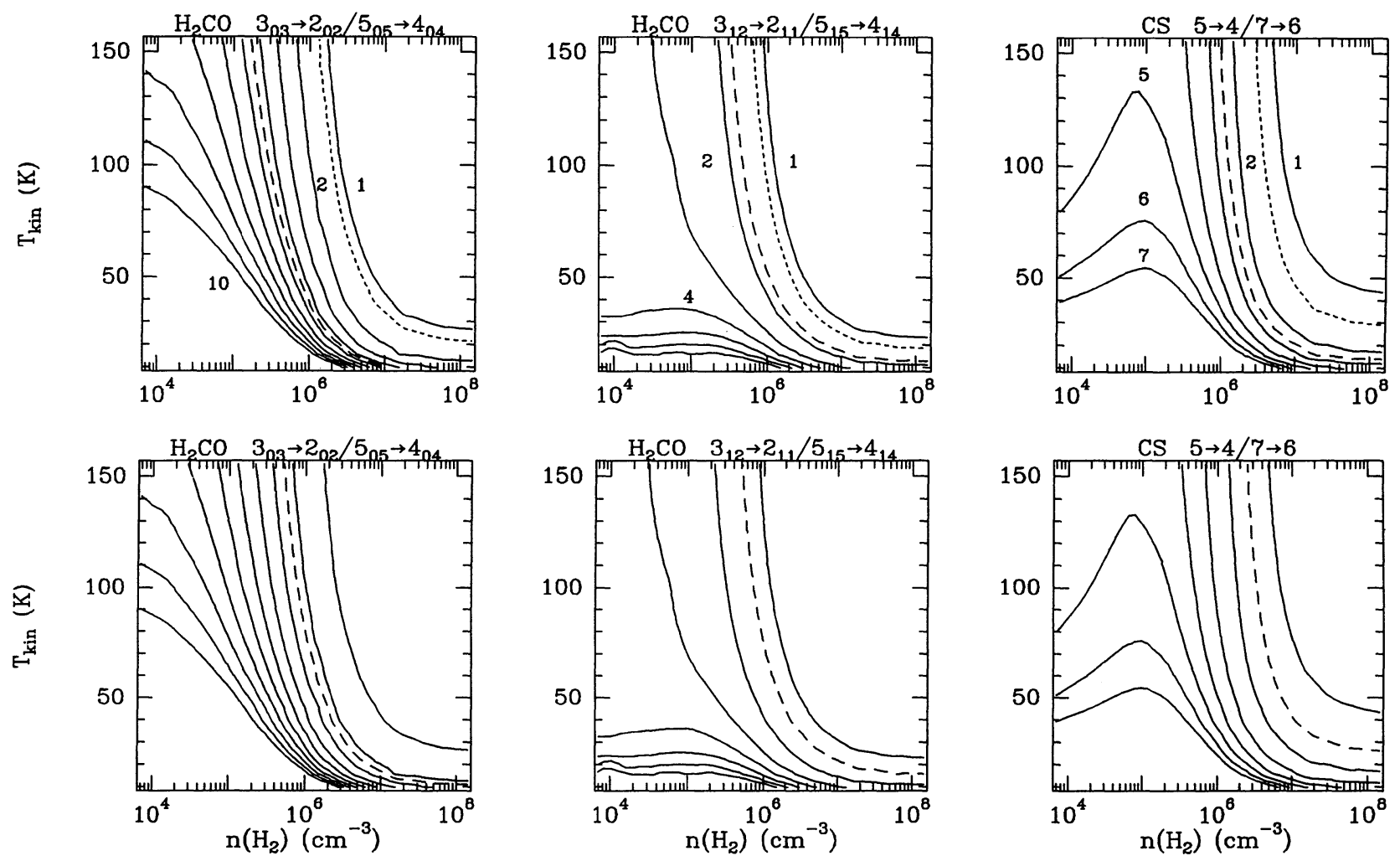

FIG. 5.-Pictorial outlines of the results of full statistical equilibrium/radiative transfer models for $\mathrm{CS}$ and $\mathrm{H}_{2} \mathrm{CO}$ transitions that are primarily sensitive to density. In each panel, the ratios of selected lines are plotted as a function of kinetic temperature and $\mathbf{H}_{2}$ volume density as variables. The observed ratios are presented as long-dashed lines for the narrow-velocity "core" component and as short-dashed lines for the wider-velocity "wing" component. The top three panels outline the results for IRAS 4A, while the bottom three panels outline the results for IRAS 4B. Plots are run for CS and $\mathrm{H}_{2} \mathrm{CO}$ column densities of $1 \times 10^{14}$ and $4 \times 10^{13} \mathrm{~cm}^{-2}$ and "core" and "wing" line widths of 1.5 and $7.5 \mathrm{~km} \mathrm{~s}^{-1}$.
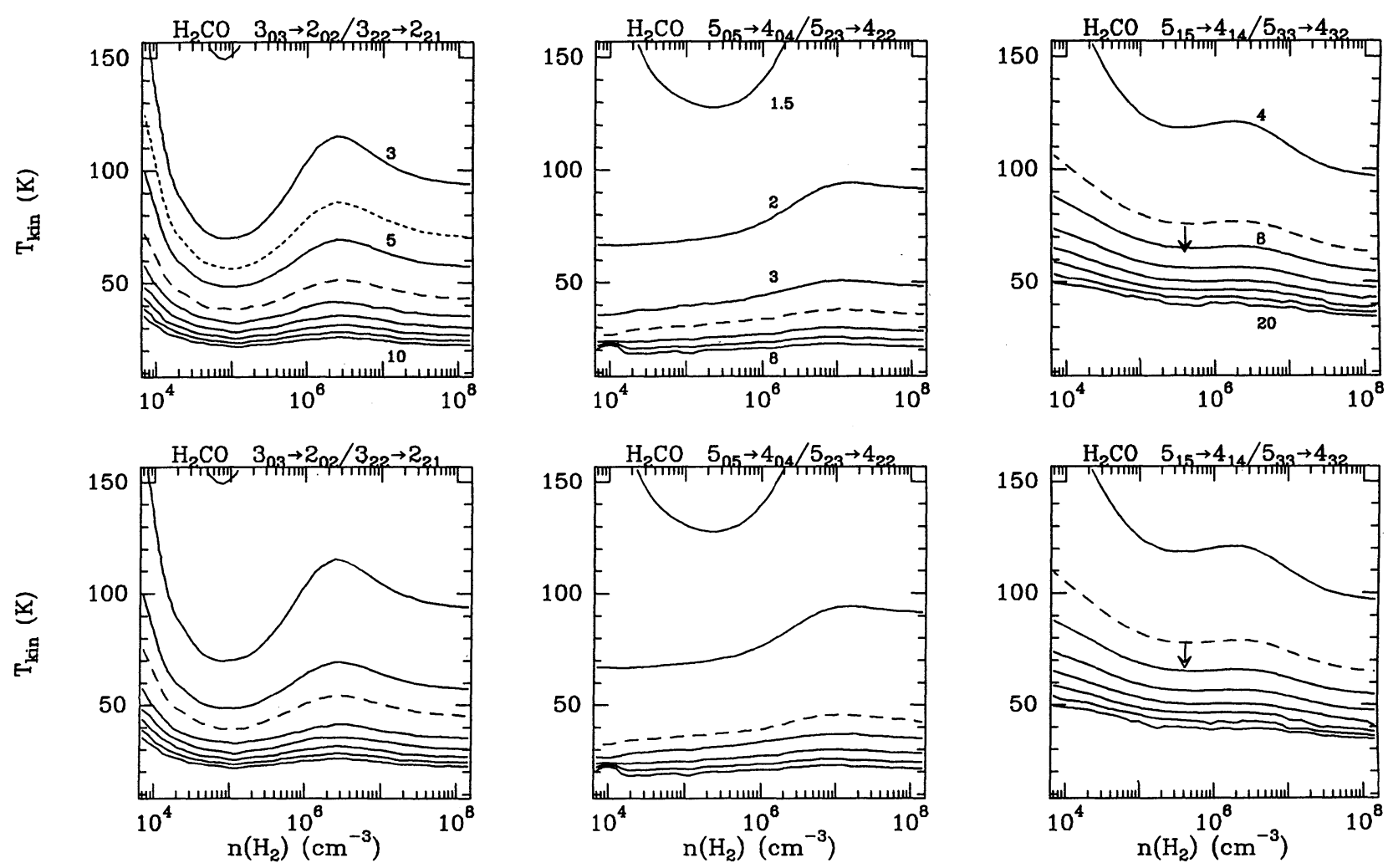

Fig. 6.-Radiative transfer plots as in Fig. 5, but now for $\mathrm{H}_{2} \mathrm{CO}$ transitions and ratios that are primarily sensitive to kinetic temperature 
TABLE 2

\begin{tabular}{|c|c|c|c|c|}
\hline \multirow[b]{3}{*}{ SPECIES } & \multicolumn{4}{|c|}{ MoleCUlar COLumn Densities ${ }^{a}$} \\
\hline & \multicolumn{2}{|c|}{ IRAS 4A } & \multicolumn{2}{|c|}{ IRAS 4B } \\
\hline & Core & Wing & Core & Wing \\
\hline $\mathrm{CO} \ldots \ldots \ldots \ldots$ & & $4.0(16)$ & & $9.0(15)$ \\
\hline${ }^{13} \mathrm{CO} \ldots \ldots \ldots$ & $1.0(16)$ & $\ldots$ & $8.5(15)$ & $\ldots$ \\
\hline $\mathrm{C}^{18} \mathrm{O} \ldots \ldots \ldots$ & $2.8(15)$ & $\ldots$ & $2.1(15)$ & $\ldots$ \\
\hline $\mathrm{C}^{17} \mathrm{O} \ldots \ldots \ldots$ & $5.3(14)$ & & $5.5(14)$ & \\
\hline $\mathrm{CS} \ldots \ldots \ldots \ldots$ & $>1.1(13)^{b}$ & $3.9(13)$ & $>6.7(12)^{b}$ & $2.6(13)$ \\
\hline$C^{34} S \ldots \ldots \ldots$ & $3.8(12)$ & & $\ldots$ & $\ldots$ \\
\hline SO $\ldots \ldots \ldots \ldots$ & $3.8(13)$ & $6.5(13)$ & $\ldots$ & $\ldots$ \\
\hline $\mathrm{SO}_{2} \ldots \ldots \ldots \ldots$ & $\ldots$ & $<4.0(13)$ & $\ldots$ & $\ldots$ \\
\hline $\mathrm{SiO}^{2} \ldots \ldots \ldots \ldots$ & & $2.8(12)$ & & \\
\hline HCN .......... & $2.2(12)$ & $1.5(13)$ & $7.5(12)$ & $8.0(12)$ \\
\hline HNC ........... & $1.4(13)^{\mathrm{c}}$ & $\ldots$ & $6.0(12)^{\mathrm{c}}$ & $\ldots$ \\
\hline DCN $. . . \ldots \ldots . .$. & $9.0(11)$ & & & $\ldots$ \\
\hline $\mathrm{HCO}^{+} \ldots \ldots \ldots$ & $>2.4(12)^{b}$ & $2.0(12)$ & $2.8(12)$ & $\ldots$ \\
\hline $\mathrm{H}^{13} \mathrm{CO}^{+} \ldots \ldots$ & $5.0(11)$ & $\ldots$ & $<1.0(11)$ & $\ldots$ \\
\hline $\mathrm{DCO}^{+} \ldots \ldots \ldots$ & $1.4(12)$ & $\ldots$ & $<2.0(11)$ & $\ldots$ \\
\hline $\mathrm{N}_{2} \mathrm{H}^{+} \ldots \ldots \ldots$ & $8.5(12)^{d}$ & $\ldots$ & $\ldots$ & $\ldots$ \\
\hline $\mathrm{H}_{2} \mathrm{D}^{+} \ldots \ldots \ldots$ & $<3.7(12)^{d}$ & . & $\ldots$ & \\
\hline $\mathrm{CH}_{3} \mathrm{OH} \ldots \ldots$ & & $1.3(15)^{\mathrm{e}}$ & & $9.3(14)$ \\
\hline $\mathrm{H}_{2} \mathrm{CO} \ldots \ldots \ldots$ & $2.6(13)$ & $7.0(13)$ & $2.6(13)$ & $3.9(13)$ \\
\hline $\mathrm{H}_{2}^{13} \mathrm{CO} \ldots \ldots$. & $3.7(12)$ & $\ldots$ & $\ldots$ & $\ldots$ \\
\hline
\end{tabular}

a From statistical equilibrium calculations at $T_{k} \approx 40-55 \mathrm{~K}$ and $n\left(\mathrm{H}_{2}\right) \approx$ $(2-4) \times 10^{6} \mathrm{~cm}^{-3}$ for the cores, and $T \approx 70-100 \mathrm{~K}$ with similar densities for the wings. All column densities in $\mathrm{cm}^{-2}$. Notation a(b) indicates $a \times 10^{b}$.

${ }^{b}$ Column density uncertain due to large optical depth.

c Core/wing partitioning uncertain due to low-velocity resolution.

d From van Dishoeck et al. 1992b.

- From rotation diagrams.

The radiative transfer/statistical equilibrium calculations also produce better estimates of the molecular column densities since the level populations are calculated selfconsistently. Table 2 presents the column densities derived for the various components toward IRAS 4A and IRAS 4B from these codes, with the exception of $\mathrm{CH}_{3} \mathrm{OH}$, for which the rotation diagram method was employed. Certain species, mostly notably $\mathrm{SiO}$, are dramatically enhanced, even in absolute terms, in the higher velocity dispersion outflow material. Others such as $\mathrm{C}^{18} \mathrm{O}$ and $\mathrm{H}^{13} \mathrm{CO}^{+}$, clearly sample the quiescent gas. The formal uncertainties in the derived column densities are generally of order $30 \%$. Some of the estimates, especially those for $\mathrm{HCN}$ and $\mathrm{HNC}$, are quite sensitive to the assumed excitation parameters since the collisional cross sections are smaller than for other species (hence the critical density is higher) or because we are observing states whose upper levels lie at or higher than the derived excitation temperatures. In addition, the distinction between the core (narrow) and wing (broad) component is not clear for HNC, since it was only observed with the $500 \mathrm{MHz}$ CSO AOS. For $\mathrm{HNC}$ and $\mathrm{HCN}$ the column densities are probably accurate only to within a factor of 2 . Observations of lower $J$ transitions at similar spatial resolution would reduce this uncertainty considerably.

\section{DISCUSSION}

Analyses of the chemical composition of YSOs ultimately require accurate estimates of the fractional abundances of molecular species, which involve a number of additional steps beyond the directly determined column densities presented in Table 2. The derivation of absolute fractional abundances requires either the assumption of some "fiducial" abundance as a standard or an estimation of the total $\mathrm{H}_{2}$ column density.
Most often, the molecular standard chosen is an optically thin isotrope of $\mathrm{CO}$, which is assumed to have "terrestrial " isotopic ratios and a $\left({ }^{12} \mathrm{C}^{16} \mathrm{O}\right) /\left(\mathrm{H}_{2}\right)$ abundance of $1 \times 10^{-4}$. $\mathrm{CO}$ is not thought to vary appreciably due to the chemical stability of $\mathrm{CO}$ and its low sublimation temperature (Black \& Willner 1984; van Dishoeck et al. 1992a). Our adopted CO abundance is somewhat lower than that observed recently toward NGC 2024 IRS 2 by Lacy et al. (1994).

An alternative estimate is provided by using the dust continuum flux to derive the beam-averaged $\mathrm{H}_{2}$ column density. Below we review the available continuum data on IRAS 4A and $4 \mathrm{~B}$ before turning to an examination of the molecular abundances in these sources.

\subsection{Dust Continuum Analyses of IRAS $4 A$ and $4 B$}

Estimates of the mass and column density can be calculated from dust flux measurements using the equations

$$
\begin{aligned}
M= & 1.90 \times 10^{-3} C_{250}\left(1.2 \times 10^{12} / v\right)^{\beta+3} S_{\mathrm{Jy}} \\
& \times\left(e^{h v / k T_{d}}-1\right) d_{\mathrm{kpc}}^{2} M_{\odot}, \\
N\left(H+H_{2}\right)= & 8.10 \times 10^{18} C_{250}\left(\frac{1.2 \times 10^{12}}{v}\right)^{\beta+3} S_{\mathrm{Jy}} \\
& \times\left(e^{h v / k T_{d}}-1\right)\left(\frac{20^{\prime \prime}}{\mathrm{FWHM}}\right)^{2} \mathrm{~cm}^{-2},
\end{aligned}
$$

where $C_{250}$ is the reciprocal of the mass opacity at $\lambda=250 \mu \mathrm{m}$ (here we adopt a value of $10 \mathrm{~g} \mathrm{~cm}^{-2}$ [Hildebrand 1983]); $v$ is the frequency in $\mathrm{Hz} ; \beta$ is the power-law index for the dust emissivity; $S_{\mathrm{Jy}}$ is the integrated flux density in janskys; $d_{\mathrm{kpc}}$ is the distance in kiloparsecs; and FWHM is the beam full width at half-maximum in arcseconds. Note that while the total mass is dependent upon $\left(d_{\mathrm{kpc}}\right)^{2}$, the derived column density is independent of distance as long as the source fills the beam. The physical density scales as $\left(d_{\mathrm{kpc}}\right)^{-1}$. Dust in moderately dense clouds is characterized by a value of $\beta=2$ (Hildebrand 1983), but recent observations at millimeter and submillimeter wavelengths suggest that $\beta \sim 1$ is more appropriate for molecular cloud cores containing young stars (Beckwith \& Sargent 1991; Weintraub, Sandell, \& Duncan 1991). Employing $\beta=1$ minimizes the mass and column density estimated from dust and so is used here.

Recent interferometric data at $\lambda=2.8$ and $\lambda=1.4 \mathrm{~mm}$ (Mundy et al. 1995) coupled with the above equations yield total mass and column density estimates of $3.5,1.5 M_{\odot}$, and $1.4 \times 10^{23}, 0.6 \times 10^{23} \mathrm{~cm}^{-2}$ for IRAS 4A, 4B over $20^{\prime \prime}$ beams. Models of the continuum emission suggest that for $\lambda \leq 1.4 \mathrm{~mm}$ the dust in the two sources may be becoming optically thick. For example, utilizing the same expressions above, but now employing the submillimeter fluxes from Sandell et al. (1991) reduces the mass estimates to $1.4 M_{\odot}$ for IRAS 4A. The millimeter wavelength continuum emission region is clearly resolved by interferometer observations and is consistent with a power-law envelope structure over the scale of our single-dish measurements. The density index is difficult to constrain, but in all cases the beam averaged physical density is $\geq 1 \times 10^{7}$ $\mathrm{cm}^{-3}$. At $1300 \mathrm{AU}$, power-law models with $n=n_{0}\left(r / r_{0}\right)^{-1.9}$ yield a density of $2 \times 10^{7} \mathrm{~cm}^{-3}$ (Mundy et al. 1995). These estimates are nearly a factor of 10 above those found for envelopes around T Tauri stars (Kenyon, Calvet, \& Hartmann 1993) but are consistent with the expected properties of extreme Class I YSOs. It is not possible from our data to put 
new constraints on the dust temperature, but for material on single-dish size scales the temperatures are unlikely to exceed the fitted global dust temperature of 33 K (Sandell et al. 1991).

\subsection{Molecular Abundances in IRAS $4 A$ and $4 B$}

The conditions outlined above apply to the core material surrounding IRAS 4A and 4B. Due to the much smaller amount of material involved, estimates for the mass of the gas and dust in the outflow must rely on the observations of $\mathrm{CO}$ described in $\S 3.1$. The difficulty associated with this scheme is illustrated in Figure 7, which shows spectra of the CO and CS main isotopic transitions at offsets of only $15^{\prime \prime}$ from the continuum position of IRAS 4A. As is typical for most outflows, the wings extend well into the nominal cloud velocity range, and it is difficult to accurately discern low-velocity outflowing gas from truly quiescent material. $\mathrm{CH}_{3} \mathrm{OH}$, for example, has line profiles that are often much narrower than those of $\mathrm{CO}$ and CS, but it too shows the velocity pattern illustrated in Figure 7. In addition, since we do not directly measure the excitation temperature of the $\mathrm{CO}$ and only have upper limits on the optical depth, the overall mass is known only to within a factor of a few. The column density calculated from the $\mathrm{CO}$ line wings in the optically thin approximation at an excitation temperature of $22 \mathrm{~K}$ is $4 \times 10^{16} \mathrm{~cm}^{-2}$, or total column density of $4 \times 10^{20} \mathrm{H}_{2} \mathrm{~cm}^{-2}$ in a $20^{\prime \prime}$ beam.

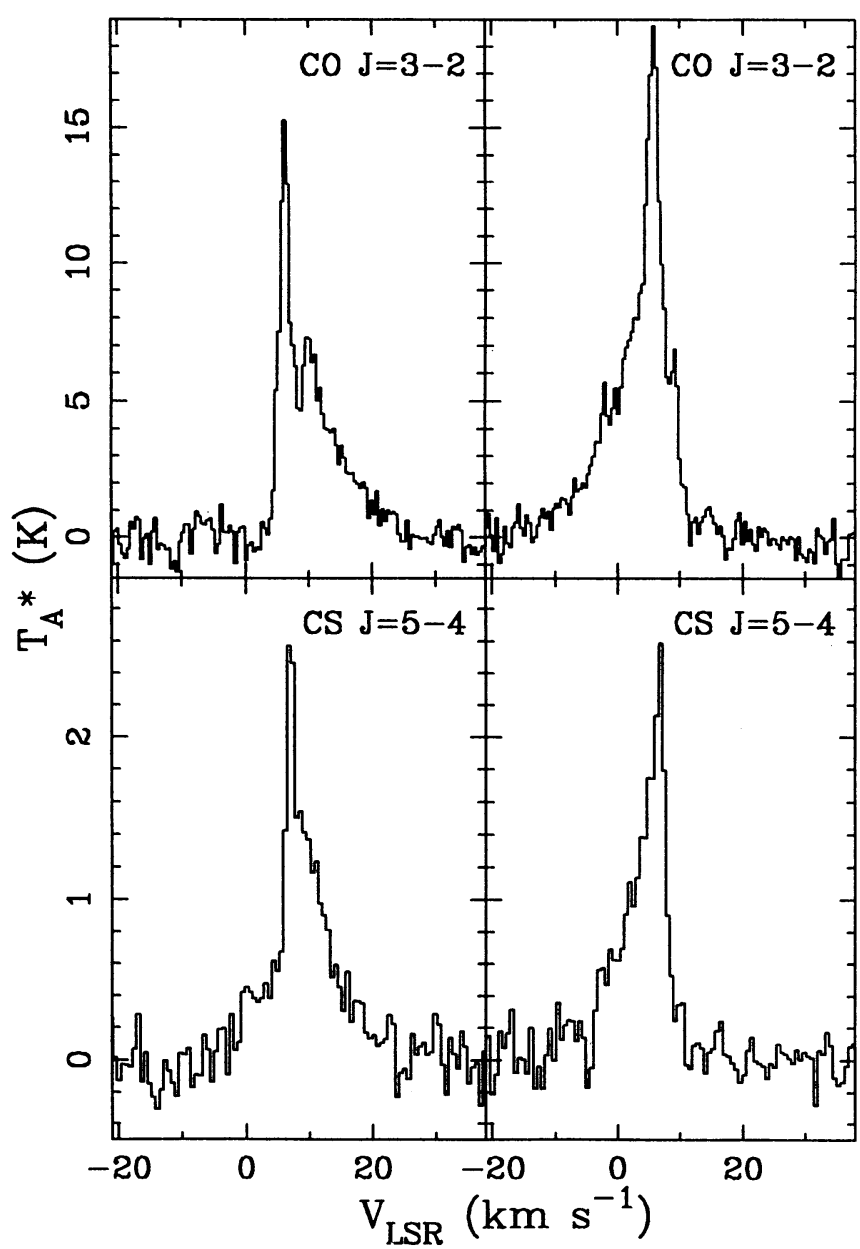

Fig. 7.-JCMT spectra of the CO $3 \rightarrow 2$ and CS $7 \rightarrow 6$ outflow wings at positions 10" NE (left) and 10" SW (right) of IRAS 4A.
For the quiescent material, observations of the $\mathrm{C}^{17} \mathrm{O}$ and $\mathrm{C}^{18} \mathrm{O}$ lines toward IRAS $4 \mathrm{~A}$ are consistent with a total $\mathrm{H}_{2}$ column density of $1.4 \times 10^{22} \mathrm{~cm}^{-2}$, an order of magnitude smaller than that derived from the dust; optical depth effects are found to be minor for both of these isotopomers. Table 3 summarizes the results of each of these different methods of estimating molecular abundances in various regions of IRAS 4, namely, dust continuum versus molecular line emission using $\mathrm{C}^{18} \mathrm{O} / \mathrm{C}^{17} \mathrm{O}$ for the core and statistical equilibrium estimates for the outflowing gas using ${ }^{12} \mathrm{CO}$ as the mass tracer.

Table 3 also presents the abundances found in two other low-mass and one high-mass star-forming clouds. Despite the large uncertainties, several dramatic trends are immediately apparent. In the core region, all of the molecular abundances are extremely low if dust is used to estimate the total column density. The derived abundance of $\mathrm{CO}$, for example, is $1 \times 10^{-5}$, at least a factor of 10 below the "canonical" value. Other molecules have abundances that are depressed by similar amounts. For species such as $\mathrm{CS}, \mathrm{CO}$, and $\mathrm{HCO}^{+}$, observations of isotopic species ensure that optical depths are not to blame.

Gas-phase depletions have also been suggested in a variety of other sources (Mauersberger et al. 1992; Blake, van Dishoeck, \& Sargent 1992; Goldsmith at al. 1992; McMullin, Mundy, \& Blake 1993), but in many cases the observational uncertainties associated with the determinations of molecular abundances along with poorly constrained dust opacity and emissivity parameters make the depletion factors highly model dependent. Here, we stress that substantial depletions are found even for parameters that minimize the column density estimates from the observed millimeter and submillimeter dust fluxes. The true depletions could therefore be even larger.

When the abundances are scaled with respect to $\mathrm{CO}$, however, the observed chemistry is similar to dark clouds such as L134N and other low-mass star-forming cores such as IRAS 16293-2422. Indeed, the abundances in IRAS 16293-2422 and IRAS 4 generally lie within a factor of a few of each other, with the exception that deuterated species appear to be somewhat enhanced in IRAS 4. The major difference between these sources is in the molecular emission strength relative to the dust continuum flux. In principle, this difference could be caused by large differences in the dust optical depth or emissivity. That is, if the dust is optically thick in IRAS 4 but optically thin in IRAS 16293 at millimeter wavelengths, or if the two sources are characterized by very different grain emissivities, this could account for the relative difference in perceived gas-to-dust ratios. Certainly dust opacity at scales of $10-100 \mathrm{AU}$ is a major issue (Beckwith et al. 1990), but the power-law envelopes observed around extreme Class I objects place most of their mass at large radius where it is very difficult to maintain $\tau_{\text {DUST }} \geq 1$ or greatly modify $\beta$ in the millimeterwave region unless substantial grain growth occurs. As noted above, selecting shorter wavelength points on the SED only drops the predicted mass by a factor of $1.5-2$, not nearly enough to account for the observed depletions.

Interestingly, the depletion factors may be quite different for neutral species and for molecular ions. To illustrate this point, Figure 8 presents spectra of the $\mathrm{HCO}^{+}$and $\mathrm{HCN} J=4 \rightarrow 3$ transitions toward IRAS $4 A$ and $4 B$. Especially toward IRAS $4 \mathrm{~A}$, the $\mathrm{HCO}^{+}$line profile is dominated by an intense, narrow $\left(\Delta V=1-1.5 \mathrm{~km} \mathrm{~s}^{-1}\right)$ core component with fairly weak outflow wings. In contrast, the $\mathrm{HCN}$ emission consists almost entirely of higher velocity dispersion, outflowing gas, with a line-width 
TABLE 3

Molecular Abundances ${ }^{a}$

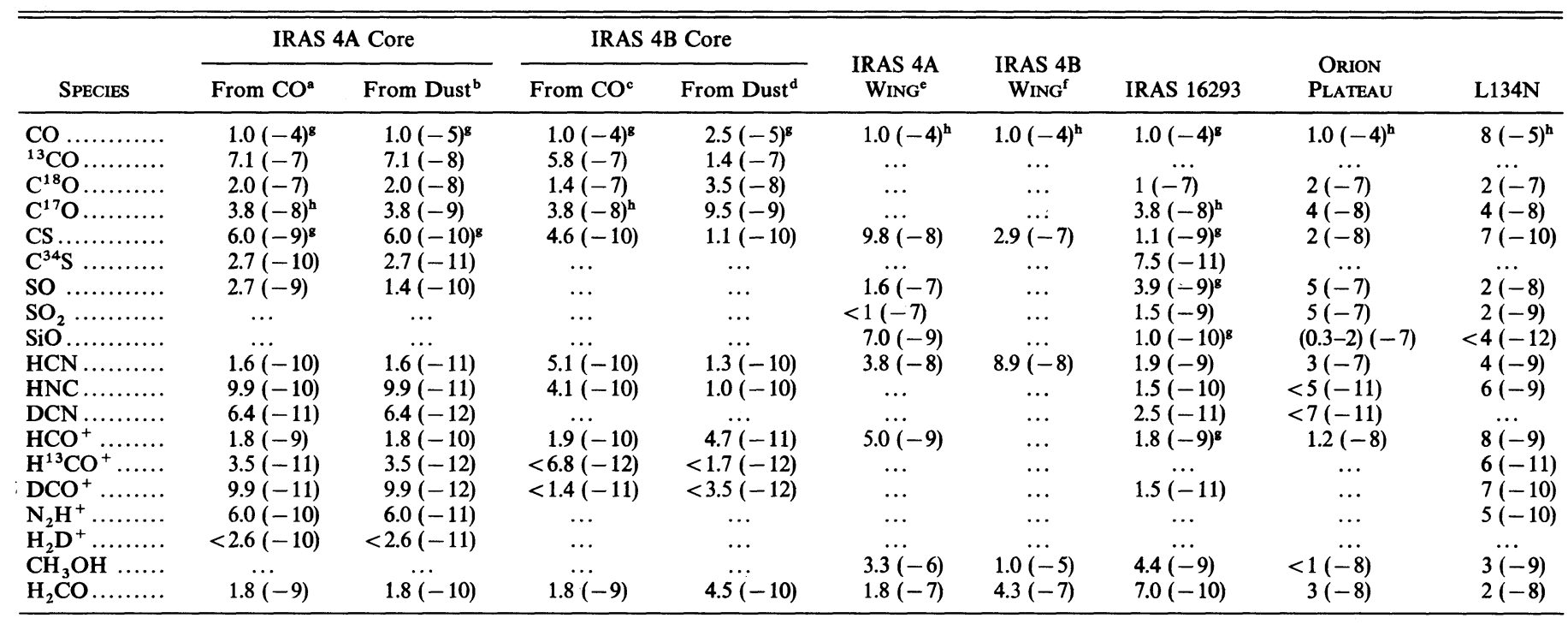

a $N\left(\mathrm{H}_{2}\right)=1.4 \times 10^{22} \mathrm{~cm}^{-2}$.

${ }^{\mathrm{b}} N\left(\mathrm{H}_{2}\right)=1.4 \times 10^{23} \mathrm{~cm}^{-2}$.

c $N\left(\mathrm{H}_{2}\right)=1.5 \times 10^{22} \mathrm{~cm}^{-2}$.

d $N\left(\mathrm{H}_{2}\right)=6.0 \times 10^{22} \mathrm{~cm}^{-2}$.

if $N\left(\mathrm{H}_{2}\right)=4.0 \times 10^{20} \mathrm{~cm}^{-2}$, assuming $\mathrm{CO} / \mathrm{H}_{2}=1 \times 10^{-4}$.

${ }^{\mathrm{f}} \mathrm{N}\left(\mathrm{H}_{2}\right)=9.0 \times 10^{19} \mathrm{~cm}^{-2}$, assuming $\mathrm{CO} / \mathrm{H}_{2}=1 \times 10^{-4}$.

${ }^{8}$ From isotopic species assuming normal isotopic abundances.

${ }^{h}$ Abundance fixed.

REFERENCES.-IRAS 16293: Blake et al. 1994, van Dishoeck et al. 1995; Orion Plateau: Blake et al. 1987; L134N: Ohishi, Irvine, \& Kaifu 1992.

rivaling that observed in $\mathrm{CO}$. The lack of strong $\mathrm{HCN}$ emission in the core could be accounted for by subthermal excitation, as the collisional cross sections for this species are smaller than those for $\mathrm{HCO}^{+}$, but the physical conditions deduced by the statistical equilibrium codes suggest this is not the case. Optical depth in the dust is also unable to explain the difference.

In the outflowing gas we can only derive estimates using $\mathrm{CO}$ as the reference tracer. Even so, many species are found to be considerably enhanced in the outflow relative to their abundances in the core calculated in the same fashion, particularly $\mathrm{SiO}, \mathrm{SO}, \mathrm{CS}, \mathrm{HCN}, \mathrm{H}_{2} \mathrm{CO}$, and $\mathrm{CH}_{3} \mathrm{OH}$. The $\mathrm{SiO}$ emission is kinematically unique in that the velocity centroid lies at -0.5 $\mathrm{km} \mathrm{s}^{-1}$, more than $7 \mathrm{~km} \mathrm{~s}^{-1}$ removed from the local standard of rest, and must arise from directly shocked material (MartinPintado, Bachiller, \& Fuente 1992; McMullin, Mundy, \& Blake 1994a). All of the other species show shifts of $\leq 1-2 \mathrm{~km}$ $\mathrm{s}^{-1}$ from the $\mathrm{C}^{18} \mathrm{O}$ derived systemic velocity of $V_{\mathrm{LSR}}=6.7 \mathrm{~km}$ $\mathrm{s}^{-1}$.

\subsection{Molecular Depletions in IRAS $4 A$ and $4 B$}

What processes can explain these trends? We suggest that, in both the quiescent core and the outflowing wings, gas-grain and grain-grain interactions play pivotal roles in determining the observed gas-phase composition.

In both regions, it is important to consider the collision times for gas-grain and grain-grain processes, which are given by

$$
\tau_{\text {gas-grain }}=\left(\alpha n_{\mathrm{gr}} \sigma_{\mathrm{gr}} v_{\text {mol }}\right)^{-1}, \quad \tau_{\text {grain-grain }}=\left(n_{\mathrm{gr}} \sigma_{\mathrm{gr}} v_{\mathrm{gr}}\right)^{-1},
$$

where $\alpha$ is the sticking coefficient for molecule-grain collisions; $v_{\text {mol }}$ is the molecular velocity; and $n_{\mathrm{gr}}, v_{\mathrm{gr}}, \sigma_{\mathrm{gr}}$ denote the number density, velocity, and collision cross section of an "average" grain. For "typical" values of the above parameters $\left(v_{\mathrm{gr}} \sim 0.1 \mathrm{~km} \mathrm{~s}^{-1}, \alpha=1\right)$, these equations reduce to $\tau_{\text {gas-grain }} \sim$ $T_{\text {grain-grain }}=2 \times 10^{9} / n_{\mathrm{H}_{2}}$ yr (Shalabiea \& Greenberg 1995). At the very high densities and low temperatures which characterize the dense cores surrounding IRAS 4A and 4B, the depletion timescales are measured in hundreds to thousands of years, considerably shorter than the inferred and observed dynamical timescales. Furthermore, if this core is indeed in the process of collapse, the velocity fields for the grains and gas are likely to differ since the grains are charged and the collapse may be magnetically mediated (Fiedler \& Mouschovias 1993; Galli \& Shu 1993). As a result, depletion processes are likely to increase in effectiveness during the collapse.

Competing with grain mantle accretion are a number of desorption processes, such as cosmic ray desorption, thermal evaporation, and grain-grain collisions. Cosmic-ray induced desorption timescales are on the order of $10^{5}$ years and are independent of density (Léger, Jura, \& Omont 1985), so such events are only important at densities below $10^{4} \mathrm{~cm}^{-3}$. Thermal evaporation timescales are strongly correlated with grain mantle binding energies, and at the temperatures characterizing the IRAS 4 quiescent material even weakly bonded species such as $\mathrm{CO}$ are not easily liberated. At very high densities, grain-grain collisions may well provide the necessary energy input to return grain mantle constituents to the gas phase either via an explosive mechanism if radical species are present in the mantle or via simple thermal desorption at collisional velocities in excess of $0.1 \mathrm{~km} \mathrm{~s}^{-1}$.

Molecular ions are expected to remain in the gas phase considerably longer, or deeper, into the collapse process and should therefore have higher abundances in the cold, quiescent 


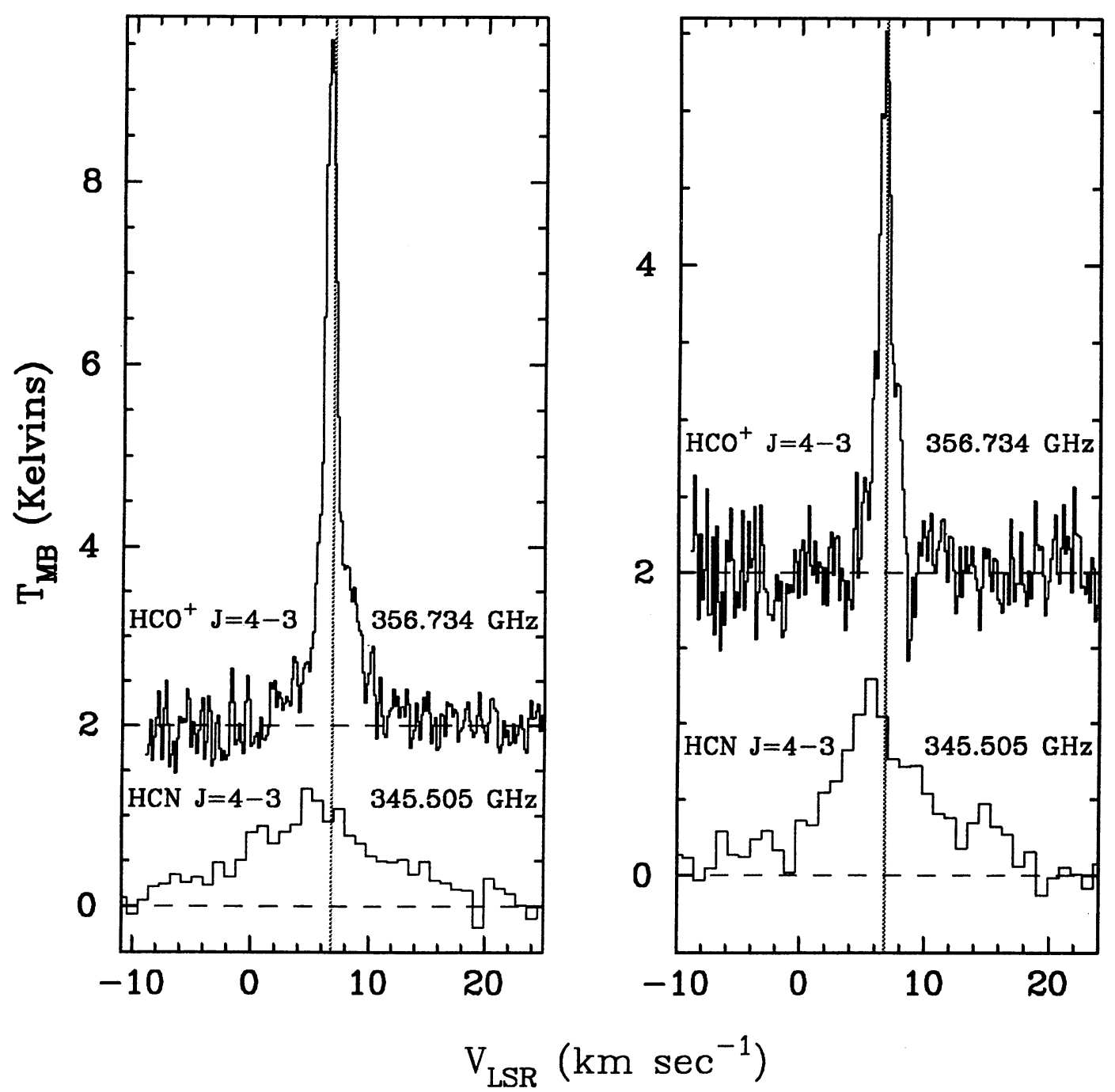

Fig. 8.-CSO spectra of the $\mathrm{HCO}^{+}$and $\mathrm{HCN} J=4 \rightarrow 3$ transitions toward IRAS $4 \mathrm{~A}$ (left) and 4B (right). Note that, despite the similar excitation requirements of these two transitions, $\mathrm{HCN}$ is largely devoid of the strong core emission present in the $\mathrm{HCO}^{+}$spectrum, while both species possess wing emission arising from the dense, outflowing gas.

gas as is illustrated in Figure 8 (see also Rawlings et al. 1992). The observed abundances suggest that molecular depletion is quite widespread, with only perhaps $5 \%-10 \%$ of the precollapse abundances remaining. In essence, only an outer "skin" of conventional abundance gas and dust remains, inside of which nearly all of the condensible material has been frozen onto the dust grain mantles (Rawlings et al. 1992). Future observations of very high density tracers such as the CS $10 \rightarrow 9$ or $\mathrm{HCO}^{+} 9 \rightarrow 8$ transitions may help to establish the physical conditions under which depletions occur.

Such extreme depletions may characterize the infall, or collapse, phase of star formation. In IRAS $4 \mathrm{~A}$ and $4 \mathrm{~B}$, the observed, redshifted absorption dips in the high-opacity ${ }^{12} \mathrm{CO}$ transitions; the red wing/blue wing asymmetry present in the moderate opacity ${ }^{13} \mathrm{CO}$ (Fig. 1), $\mathrm{HCO}^{+}$(Fig. 8), and $\mathrm{H}_{2} \mathrm{CO}$ lines, and the symmetry of the optically thin $\mathrm{C}^{\mathbf{1 8}} \mathrm{O}$ transitions are all consistent with the patterns expected for infalling envelopes around low-mass protostars (Zhou et al. 1993). The observed asymmetries are also consistent with infall rates required to provide the calculated system luminosity. Similar profiles are seen toward other young, "protostellar" sources
(Zhou et al. 1993; Chandler \& Sargent 1993; van Langevelde, van Dishoeck, \& Blake 1994). A more detailed analysis of the available single-dish and interferometer data will be presented elsewhere (Mundy et al. 1995), but we stress here that without a careful consideration of both the chemical and physical properties of various molecular tracers, it is likely that outflowing gas will confuse these kinematic infall signatures even in the closest star-forming regions.

\subsection{Chemical Modifications Driven by the Young Stellar Outflows}

It is clear from the present data set that the outflowing gas in IRAS 4 is chemically very distinct despite its similar physical state. What is driving this difference? If the jet-driven models of molecular outflows are correct, there will be an extensive "shear region" along the axis of the outflow with a wide range of shock velocities (Masson \& Chernin 1993). Within the shear region are localized zones of high compression and excitation where the extremely high velocity jets shock and directly entrain material, leading to chemical sputtering of dust grains and substantial kinetic temperatures (Jones et al. 1995). These 
regions are responsible for the hot $\mathrm{H}_{2}$ now commonly observed in outflow sources near $2 \mu \mathrm{m}$ (Davis et al. 1993; Bally, Lada, \& Lane 1993; van Langevelde et al. 1994) and may also be responsible for the observed emission from very refractory species such as $\mathrm{SiO}$ that often show both large kinematic offsets and different morphologies from bulk molecular cloud tracers (Guilloteau et al. 1992; Martín-Pintado et al. 1992; Mikami et al. 1992; McMullin et al. 1993, 1994a).

Within the more gently compressed and accelerated regions that are responsible for the widely observed $\mathrm{CO}$ wings from YSOs, the derived kinetic temperature (from measurements of CO line ratios) is typically rather cold, on the order of 10-20 $\mathrm{K}$-especially in low-luminosity objects such as IRAS 4 (Chernin \& Masson 1991; Fukui et al. 1993). Here we find similar temperatures, on the order of $15-20 \mathrm{~K}$ for $\mathrm{CH}_{3} \mathrm{OH}$ and perhaps $40-50 \mathrm{~K}$ for $\mathrm{H}_{2} \mathrm{CO}$ and $\mathrm{CS}$. These values are far too small to induce extensive thermal desorption from dust grain mantles, but the observed velocity dispersions are clearly substantial enough to induce large temperature fluctuations after grain-grain collisions. Collisional velocities of $0.5 \mathrm{~km} \mathrm{~s}^{-1}$, for example, are more than adequate to release all mantle material thermally, explosive desorption via radical runaway need not be invoked (J. Greenberg, private communication). These velocities are not sufficient to mechanically heat the gas, however, and once desorbed the warm molecules are rotationally and vibrationally thermalized by collisions with $\mathrm{H}_{2}$ very rapidly (only a handful of collisions are required). Redepletion timescales are then on the order of thousands of years, now quite comparable to the estimated dynamical times.

The large abundances of methanol, formaldehyde, hydrogen cyanide, etc., in the outflowing gas around IRAS 4A and 4B may therefore reflect their large abundance in grain mantles. We note that very high abundances of cold methanol have also been found in other low-luminosity outflow systems, most notably that of the nearby IRAS 2 bow shock (Sandell et al. 1994), and are essentially impossible to reproduce with purely gas-phase models. Coupled gas-grain models, however, can produce large amounts of methanol and formaldehyde (Brown \& Charnley 1990; Shalabiea \& Greenberg 1995), and methanol is a major grain mantle constituent toward embedded infrared sources such as W33A and W3 IRS 5 (Skinner et al. 1992; Allamandola et al. 1992). Methanol abundances as high as $10^{-5}$ are found observationally and theoretically, and recently observed grain mantle processes form a natural explanation for the abundances observed in many objects. For example, Blake et al. (1991) and Sandford \& Allamandola (1993) have found that when amorphous methanol-rich ices are heated to approximately $120 \mathrm{~K}$, type II clathrate hydrate formation traps methanol in a 17:1 $\mathrm{H}_{2} \mathrm{O}: \mathrm{CH}_{3} \mathrm{OH}$ ratio, while smaller molecules such as $\mathrm{CO}$ and $\mathrm{CO}_{2}$ can be incorporated into smaller cage sites at abundance levels of $\leq 14 \%$ that of water. Excess methanol is placed into a second phase of high volatility at $120 \mathrm{~K}$ and rapidly sublimes (Blake et al. 1991). Other species and the remnant methanol can then only be desorbed at substantially higher temperatures.

In this scenario, it is possible to have regions in which the grain mantle sublimation producis consist largely of methanol alone or of methanol plus a mixture of less volatile species, depending on the peak temperatures and durations of grain mantle heating events. The observed enhancements of several species, not simply methanol, suggests that the transient heating in IRAS 4 is sufficiently intense to release a large fraction of the grain mantles. After desorption into the gas phase, ion-molecule reactions and readsorption onto grain surfaces can drop the abundances toward their observed values. The desorbed methanol and formaldehyde can lead in turn to more complex molecules such as dimethyl ether and methyl formate via a series of ion-molecule reactions (Blake et al. 1987; Millar, Herbst, \& Charnley 1991), but the low excitation temperature, small rotational constants, and high frequencies of the present surveys render the current upper limits on these species well above predicted values.

Thus, over the size scales associated with our single-dish measurements are regions in which the local abundances of molecules vary by several orders of magnitude. Close to the YSOs at velocities near the systemic value we expect there to be substantial evidence for gas-phase molecular depletions, while over a wider range of velocities there should be evidence for outflow-induced alterations. We stress that in the above models the outflow-driven shear zones need not demonstrate clear evidence of shock chemistry. Truly shocked gas may well be very localized and difficult to detect with single-dish instruments except in very sensitive tracers such as $\mathrm{SiO}$. Indeed, with gentle enough acceleration such shear zones might become quite extended in size and appear similar in composition to the so-called hot cores associated with high-mass star-forming regions and ultracompact $H$ il regions (Walmsley 1992) or the "compact ridge" source in Orion KL (Blake et al. 1987), but with substantially lower kinetic temperatures. Clearly, future observations should be designed at very high angular and spectral resolution which can probe the details of accretionary and outflow processes at 100-1000 AU size scales.

\section{SUMMARY}

Detailed molecular line observations of the extreme Class I sources L1450/NGC 1333 IRAS 4A and 4B have confirmed them to be unusual systems. The dynamical times of the highly collimated outflow and the extraordinary continuum fluxes are both suggestive of a very early state of evolution. Dynamical masses in the outflow lobes are similar to those found in other deeply embedded, low-luminosity YSOs and show clumpy structure whose symmetry is best understood by temporal variability in the mass-loss rate or wind velocity and not from inhomogeneities in the surrounding molecular cloud.

The outflows are visible in a wide range of molecular tracers, most of which show quite large abundance enhancements. Some of these species, e.g., SiO, are refractory and must be produced in strongly shocked material in localized regions. Others, in particular formaldehyde and methanol, are quite fragile and volatile and would not survive the passage of a strong shock. Their rotational temperatures are very cold as well. We suggest that "shear zones" surrounding the highvelocity outflow core are responsible for this emission. In these regions, the higher velocity dispersion and density lead to enhanced grain-grain collision rates which desorb the grain mantles via radial runaway or induce thermal desorption if the grain-grain collision velocities exceed $0.5 \mathrm{~km} \mathrm{~s}^{-1}$. Both mechanisms can transiently evaporate nearly the entire grain mantle without substantially altering the gas kinetic temperature or accelerating it to high velocities. Future observations of molecular outflows at interferometric spatial resolution can test this hypothesis - such observations should reveal different morphologies and velocity fields for strong shock tracers $(\mathrm{SiO})$ as compared with more volatile species (e.g., $\mathrm{CH}_{3} \mathrm{OH}$ ).

Molecular emission from the cores surrounding the YSOs is surprisingly weak. Indeed, depletion factors of at least 10-20 
are derived for all molecules including CO. Unless substantial dust opacity or unusually large millimeter-wave dust emissivities can be invoked over single-dish size scales, these depletions cannot arise as an observational or modeling artifact; they are illustrative of the dominant role grain mantle depletion and desorption plays in the chemistry associated with the collapse phase of star formation. More realistic analyses of the depletion versus dust opacity/emissivity issue must involve additional high spatial and spectral resolution observations, to be modeled with detailed temperature, density, and velocity radial profiles coupled to extensive radiative transfer and excitation calculations. Such efforts are currently underway, but even now it is clear that owing to the high densities and low temperatures associated with extreme Class I YSOs, gas-grain and grain-grain interactions must be explicitly included in models of the chemistry associated with these youngest of stars.

G. A. B. would like to acknowledge support from NASA (NAGW-2297) as well as the David and Lucille Packard and Alfred P. Sloan Foundations, which work on astrochemistry in Leiden is supported by a PIONIER grant from the Netherlands Organization for Scientific Research (NWO). L. G. M. acknowledges support from NASA (NAGW-3066). The authors would also like to thank M. Greenberg and S. Sandford for helpful discussions on grain mantle processing.

\section{REFERENCES}

Adams, F. C., Lada, D. J., \& Shu, F. H. 1987, ApJ, 312, 788

Allamandola, L. J., Sandford, S. A., Tielens, A. G. G. M., \& Herbst, T. M. 1992 ApJ, 399, 134

André, P., \& Montmerle, T. 1994, ApJ, 420, 837

André, P., Ward Thompson, D., \& Barsony, M. 1993, ApJ, 406, 122

Bally, J., Lada, E. A., \& Lane, A. P. 1993, ApJ, 418, 322

Beckwith, S., \& Sargent, A. I. 1991, ApJ, 381, 250

Beckwith, S., Sargent, A. I., Chini, R., \& Güsten, R. 1990, AJ, 99, 924

Black, J. H., \& Willner, S. P. 1984, ApJ, 279, 673

Blake, D., Allamandola, L. J., Sandford, S., Hudgins, D., \& Freund, F. 1991, Science, 254, 548

Blake, G. A., Sutton, E. C., Masson, C. R., \& Phillips, T. G. 1987, ApJ, 315, 621

Blake, G. A., van Dishoeck, E. F., Jansen, D. J., Groesbeck, T. D., \& Mundy, L. G. 1994, ApJ, 428, 680

Blake, G. A., van Dishoeck, E. F., \& Sargent, A. I. 1992, ApJ, 391, L99

Brown, P. D., \& Charnley, S. B. 1990, MNRAS, 244, 432

Calvet, N., Hartmann, L., Kenyon, S. J., \& Whitney, B. A. 1994, ApJ, 434, 330

Caselli, P., Hasegawa, T. I., \& Herbst, E. 1993, ApJ, 408, 548

Cernis, K. 1990, AP\&SS, 166, 315

Chandler, C. J., \& Sargent, A. I. 1993, ApJ, 414, L29

Chernin, L. M., \& Masson, C. R. 1991, ApJ, 382, L93

Davis, C. J., Dent, W. R., Matthews, H., Aspin, C., \& Lightfoot, F. 1993 MNRAS, 266, 933

d'Hendecourt, L. B., Allamandola, L. J., \& Greenberg, J. M. 1985, A\&A, 152 130

Fiedler, R. A., \& Mouschovias, T. C. 1993, ApJ, 415, 680

Fukui, Y., Iwata, T., Mizuno, A., Bally, J., \& Lane, A. P. 1993, Protostars \&

Planets III, ed. E. H. Levy \& J. Lunine (Tucson: Univ. Arizona Press), 603

Galli, D., \& Shu, F. H. 1993a, ApJ, 417, 220 1993b, ApJ, 417, 243

Goldsmith, P. F., Margulis, M., Snell, R. L., \& Fukui, Y. 1992, ApJ, 385, 522

Groesbeck, T. D. 1994, Ph.D. thesis, California Inst. of Technology

Guilloteau, S., Bachiller, R., Fuente, A., \& Lucas, R. 1992, A\&A, 265, L49

Hartquist, T. W., Rawlings, J. M. C., Williams, D. A., \& Dalgarno, A. 1993, QJRAS, 34, 213

Helmich, F. P., Jansen, D. J., de Graauw, T., Groesbeck, T. D., \& van Dishoeck, E. F. 1994, A\&A, 283, 626

Herbig, G. H., \& Jones, B. F. 1983, AJ, 88, 1040

Hildebrand, R. H. 1983, QJRAS, 24, 267

Jansen, D. J., van Dishoeck, E. F., \& Black, J. H. 1993, A\&A, 282, 605

Jennings, R. E., Cameron, D. H. M., Cudlip, W., \& Hirst, C. J. 1987, MNRAS 226,461

Jones, A. P., Tielens, A. G. G. M., Hollenbach, D. J., \& McKee, C. F. 1995, ApJ, 433,797

Kenyon, S. J., Calvet, N., \& Hartmann, L. 1993, ApJ, 414, 676

Knee, L. B. G., et al. 1995, in preparation

Kutner, M. L., \& Ulich, B. L. 1981, ApJ, 250, 341

Lacy, J. H., Knacke, R., Geballe, T. R., \& Tokunaga, A. T. 1994, ApJ, 428, L69

Lada, C. J. 1991, in The Physics of Star Formation and Early Stellar Evolution, ed. C. J. Lada \& N. D. Kylafis (Dordrecht: Kluwer), 329
Léger, A., Jura, M., \& Omont, A. 1985, A\&A, 144, 147

Levy, E. H., \& Lunine, J., ed. 1993, Protostars \& Planets III (Tucson: Univ. Arizona Press)

Martín-Pintado, J., Bachiller, R., \& Fuente, A. 1992, A\&A, 254, 315

Masson, C. R., \& Chernin, L. M. 1993, ApJ, 414, 230

Mauersberger, R., Wilson, T. L., Metzger, P. G., Gaume, R., \& Johnston, K. J. 1992, A\&A, 256, 640

McMullin, J. P., Mundy, L. G., \& Blake, G. A. 1993, ApJ, 405, 599

1994a, ApJ, 437, 305

McMullin, J. P., Mundy, L. G., Wilking, B. A., Hezel, T., \& Blake, G. A. 1994b ApJ, 424, 222

Mikami, H., Umemoto, T., Yamamoto, S., \& Saito, S. 1992, ApJ, 392, L87

Millar, T. J., Herbst, E., \& Charnley, S. B. 1991, ApJ, 369, 147

Mundy, L. G., Wootten, H. A., Wilking, B. A., Blake, G. A., \& Sargent, A. I. 1992, ApJ, 385, 306

Mundy, L. G., et al. 1995, in preparation

Ohishi, M., Irvine, W. M., \& Kaifu, N. 1992, IAU Symp. 150, Astrochemistry of Cosmic Phenomena, ed. P. D. Singh (Dordrecht: Kluwer), 171

Poynter, R. L., \& Pickett, H. M. 1984, A Submillimeter, Millimeter, and Microwave Spectral Line Catalog (NASA JPL Publ. 80-23, Revision 2)

Raga, A. C., \& Cabrit, S. 1993, A\&A, 278, 267

Rawlings, J. M. C., Hartquist, T. W., Menten, K. M., \& Williams, D. A. 1992 , MNRAS, 255, 471

Sandell, G., \& Aspin, C. A. 1993, The JCMT Newsletter, No. 1, 43

Sandell, G., Aspin, C. A., Duncan, W. D., Russell, A. P., \& Robson, E. I. 1991 ApJ, 376, L17

Sandell, G., Knee, L. B., Aspin, C. A., Robson, E. I., \& Russell, A. P. 1994 A\&A, 285, L1

Sandford, S., \& Allamandola, L. J. 1993, ApJ, 417, 815

Shalabiea, O. M., \& Greenberg, J. M. 1995, A\&A, submitted

Shu, F. H., Najita, J., Galli, D., Ostriker, E., \& Lizano, S. 1993, in Protostars \& Planets III, ed. E. H. Levy \& J. Lunine (Tucson: Univ. Arizona Press), 3

Skinner, C. J., Tielens, A. G. G. M., Barlow, M. J., \& Justtanont, K. 1992, ApJ, 399,79

Terebey, S., Vogel, S. N., \& Myers, P. C. 1992, ApJ, 390, 181

van Dishoeck, E. F., Blake, G. A., Jansen, D. J., \& Groesbeck, T. D. 1995, ApJ, submitted

van Dishoeck, E. F., Glassgold, A. E., Guélin, M., Jaffe, D. T., Neufeld, D. A., Tielens, A. G. G. M., \& Walmsley, C. M. 1992a, in IAU Symp. 150, Astrochemistry of Cosmic Phenomena, ed. P. D. Singh (Dordrecht : Kluwer), 285

van Dishoeck, E. F. Jansen, D. J. \& Phillips, T. G. 1993, A\&A, 279, 54

van Dishoeck, E. F., Phillips, T. G., Keene, J., \& Blake, G. A. 1992b, A\&A, 261 L13

van Langevelde, H. J., van Dishoeck, E. F., \& Blake, G. A. 1994, ApJ, 425, L45

Walmsley, C. M. 1992, in Chemistry and Spectroscopy of Interstellar Molecules, ed. D. K. Bohme et al. (Tokyo: Univ. of Tokyo Press)

Weintraub, D. A., Sandell, G., \& Duncan, W. D. 1991, ApJ, 382, 270

Zhou, S. D., Evans, N. J., Kömpe, C., \& Walmsley, C. M. 1993, ApJ, 404, 232 